
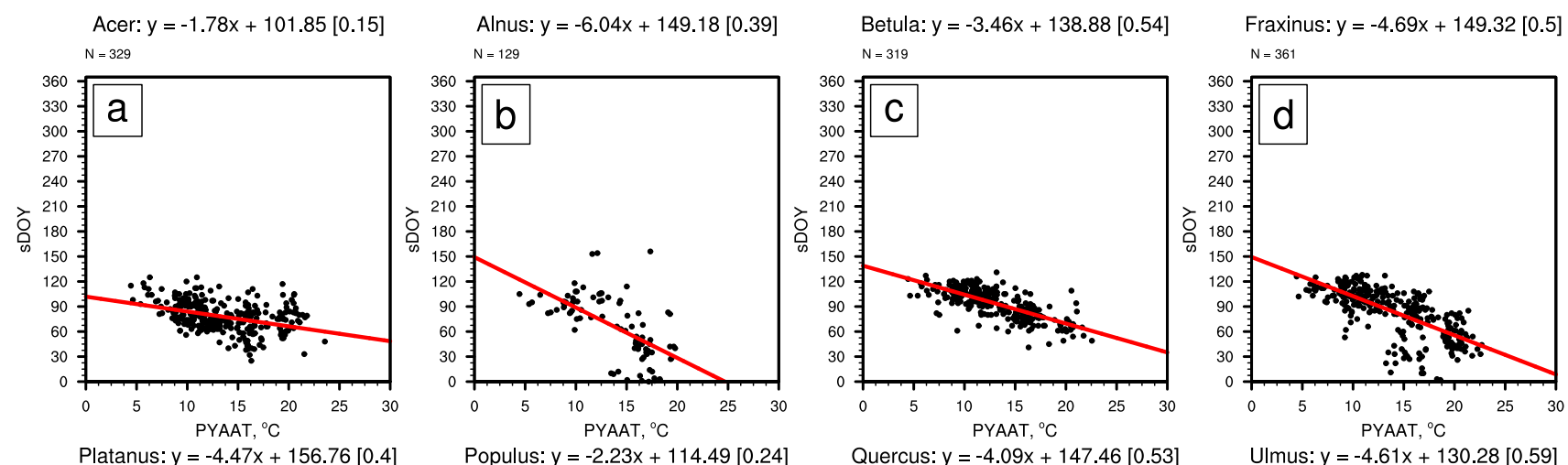

Morus: $y=-4 x+153.99[0.53]$
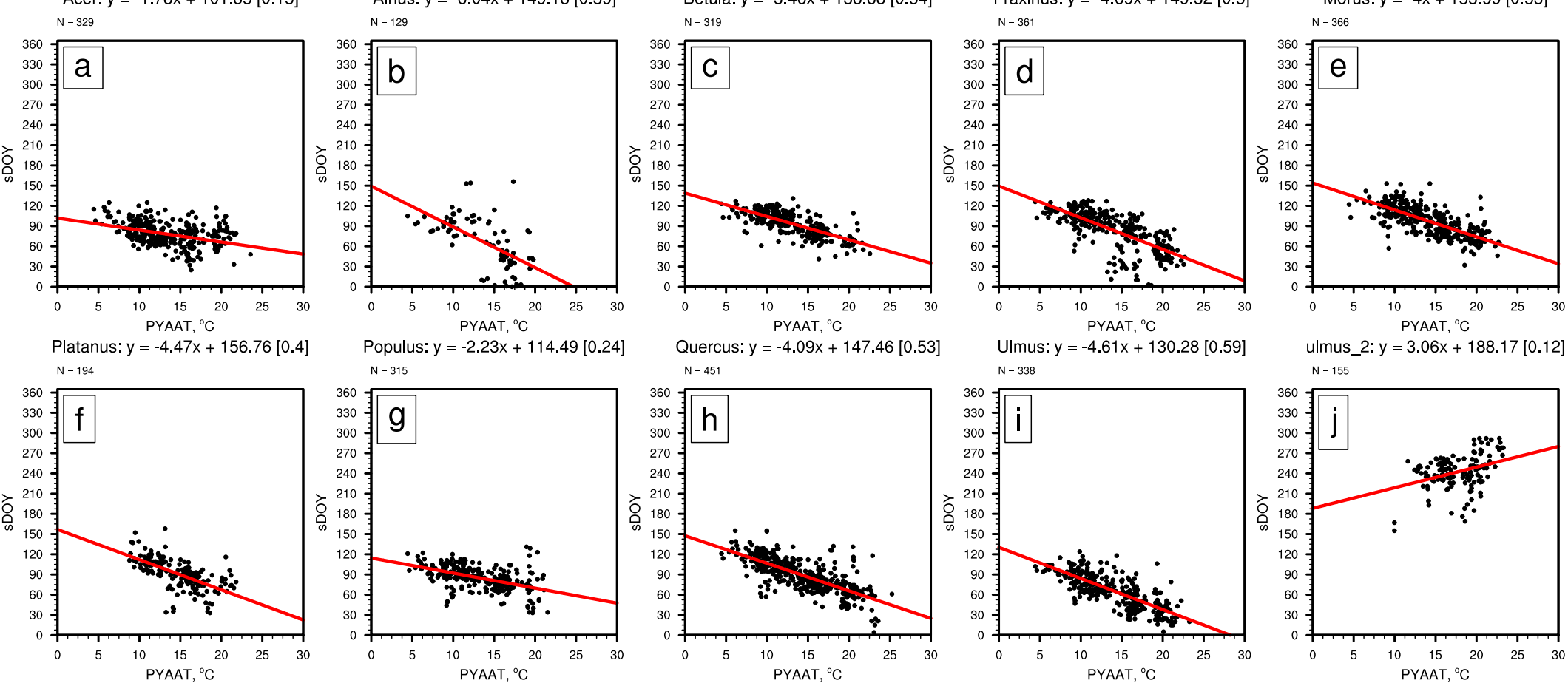

Cupressaceae: $y=-5.67 x+135.77[0.48] \quad$ Pinaceae: $y=-5.72 x+176.49[0.45]$
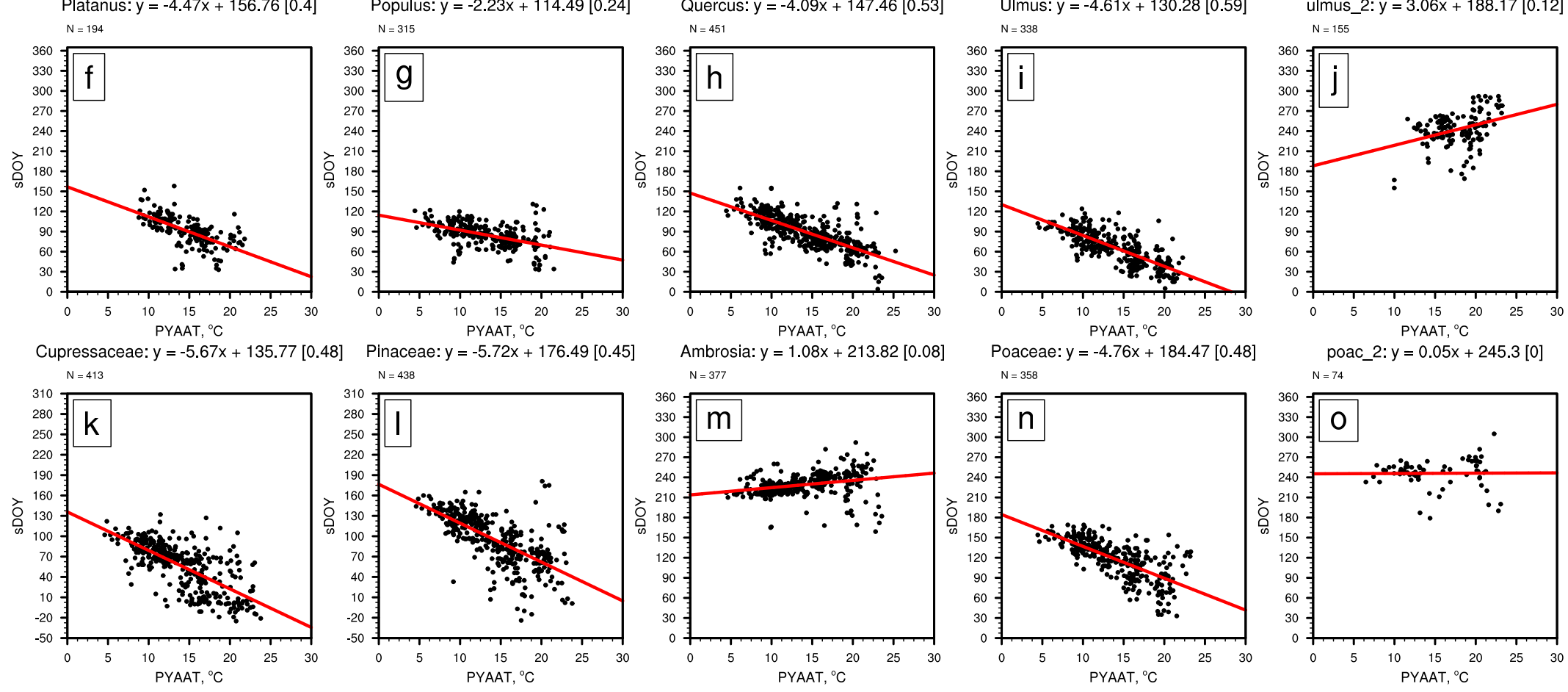

Figure S1. Linear regressions of the phenological relationship between start day-of-year (sDOY) and previous year annual average temperature 

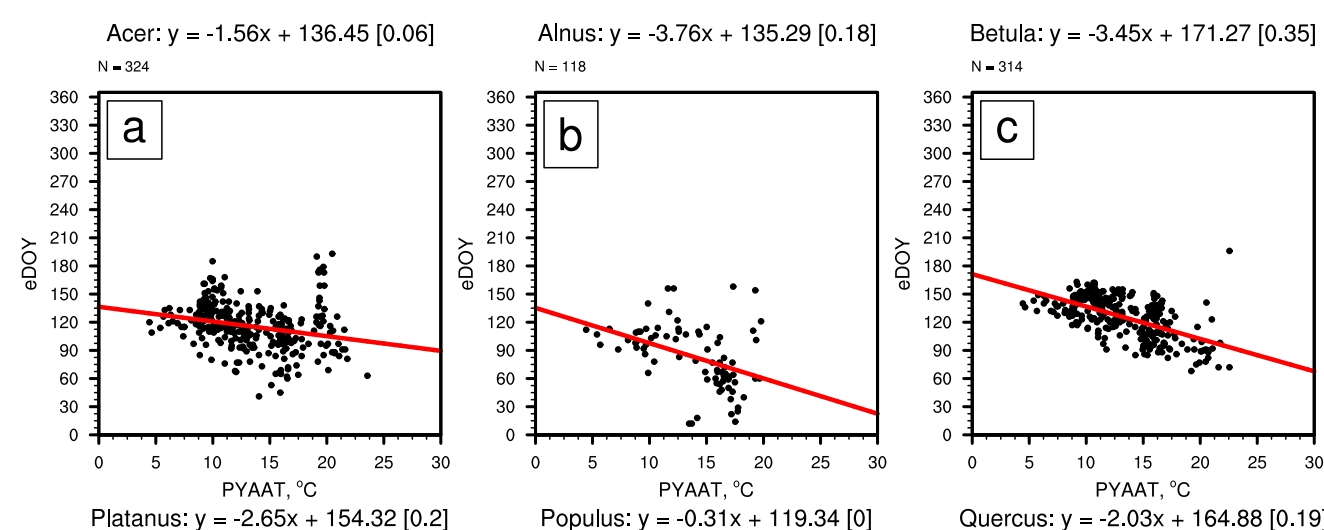

Fraxinus: $y=-2.92 x+155.62[0.32]$

Morus: $y=-2.97 x+173.67[0.29]$ $N=191$

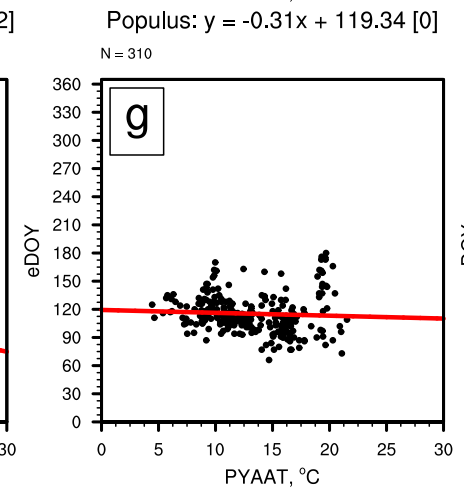

Quercus: $y=-2.03 x+164.88[0.19]$
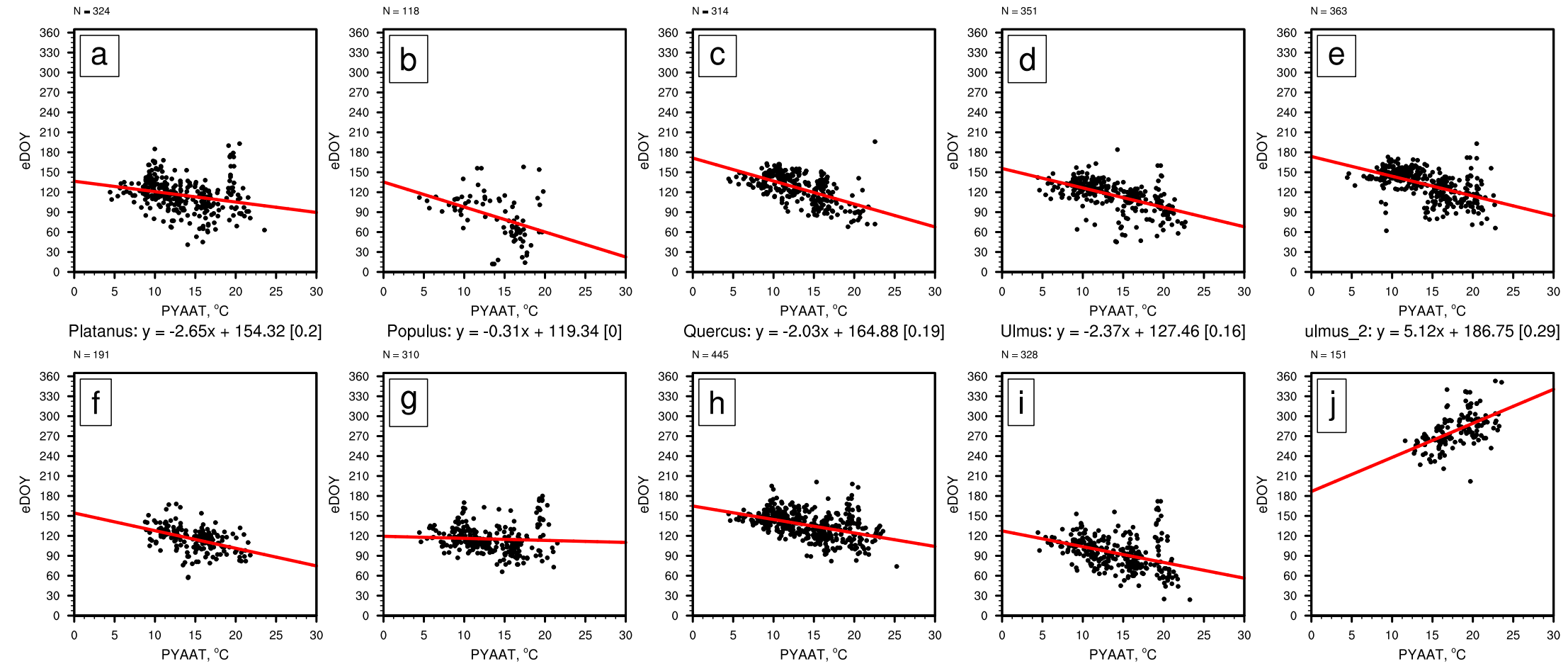

Ulmus: $y=-2.37 x+127.46[0.16]$

ulmus_2: $y=5.12 x+186.75[0.29]$
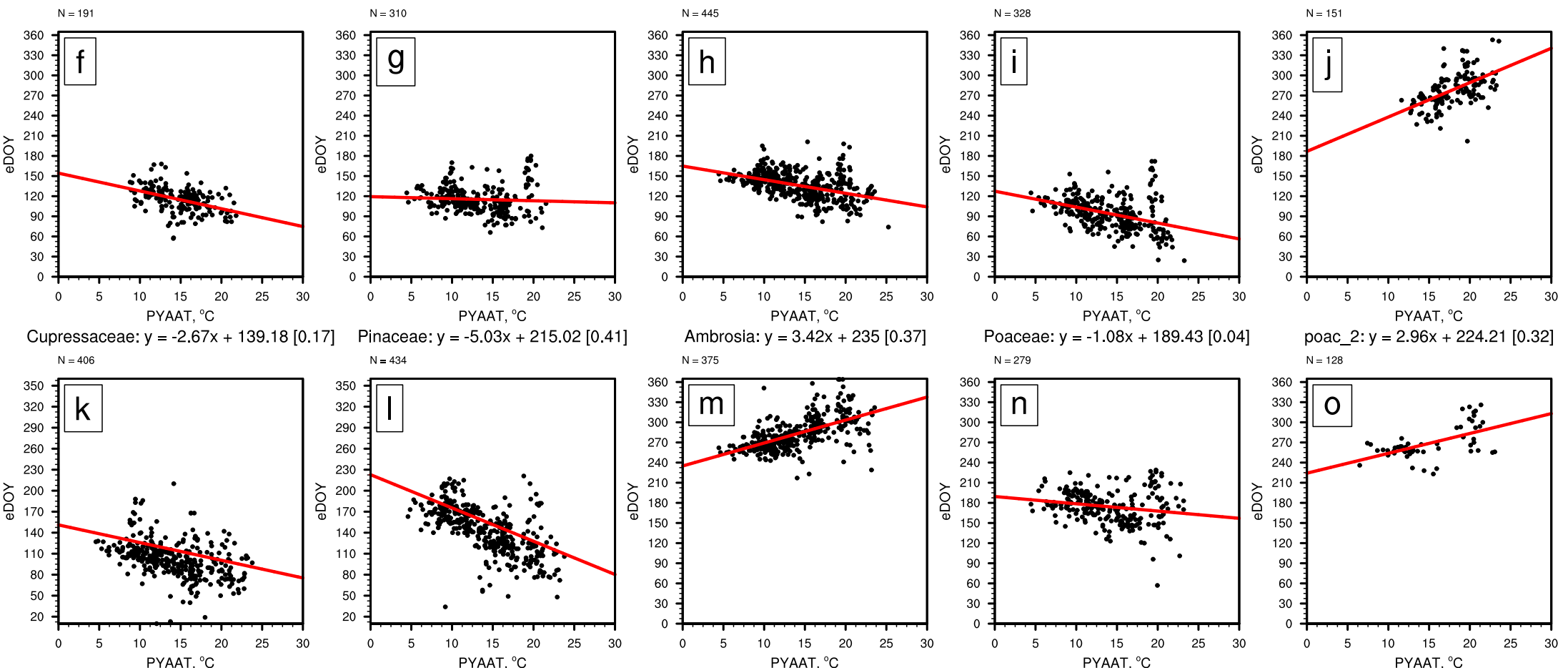

poac_2: $y=2.96 x+224.21[0.32$
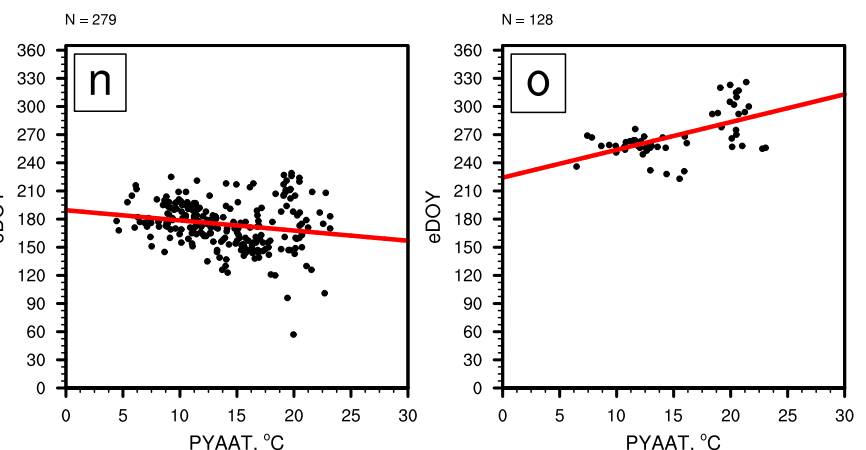

Figure S2. Same as Figure S1, but for end day-of-year (eDOY) versus PYAAT. 


\begin{tabular}{|c|c|c|c|c|c|}
\hline No. & City, State & $\begin{array}{l}\text { Latit } \\
\text { ude }\end{array}$ & $\begin{array}{l}\text { Longitu } \\
\text { de }\end{array}$ & Years & Acknowledgements \\
\hline \multicolumn{6}{|c|}{ Northeast (38) } \\
\hline 1 & Albany, NY & 42.67 & -73.80 & $2003-2010$ & $\begin{array}{l}\text { David Shulan, M.D. } \\
\text { Certified Allergy Consultants } \\
\text { Albany, NY }\end{array}$ \\
\hline 2 & Armonk, NY & 41.13 & -73.71 & $2003-2010$ & $\begin{array}{l}\text { Guy Robinson, PhD } \\
\text { The Louis Calder Center } \\
\text { Armonk, NY }\end{array}$ \\
\hline 3 & Baltimore, MD & 39.30 & -76.61 & $2003-2010$ & $\begin{array}{l}\text { Jonathon Matz, MD FAAAAI \& } \\
\text { David Golden, MD FAAAAI } \\
\text { Dr. Golden and Dr. Matz, LLC }\end{array}$ \\
\hline 4 & Boston, MA & 42.35 & -71.06 & 2010 & $\begin{array}{l}\text { Immunology Research Institute of New England } \\
\text { Lawrence M. DuBuske, MD FAAAAI } \\
\text { Boston, MA }\end{array}$ \\
\hline 5 & Brooklyn, NY & 40.65 & -73.95 & $2004-2010$ & $\begin{array}{l}\text { Clifford W. Bassett, MD \& Mehdi Vesaghi, MD } \\
\text { Long Island College Hospital } \\
\text { Brooklyn, NY }\end{array}$ \\
\hline 6 & Chelmsford, MA & 42.60 & -71.37 & $2003-2005$ & $\begin{array}{l}\text { Julian Melamed, M.D. } \\
\text { Chelmsford, MA }\end{array}$ \\
\hline
\end{tabular}




\begin{tabular}{|c|c|c|c|c|c|}
\hline 7 & Cherry Hill, NJ & 39.90 & -75.00 & $2003-2010$ & $\begin{array}{l}\text { Larchment Medical Center II } \\
\text { Donald J. Dvorin, MD FAAAAI } \\
\text { Cherry Hill, NJ }\end{array}$ \\
\hline 8 & Chicago, IL & 41.84 & -87.68 & $2003-2010$ & $\begin{array}{l}\text { John Shane, PhD } \\
\text { McCrone Research Institute } \\
\text { Chicago, IL }\end{array}$ \\
\hline 9 & Dayton, $\mathrm{OH}$ & 39.78 & -84.20 & $2003-2010$ & $\begin{array}{l}\text { Mr. Andy Roth } \\
\text { RAPCA } \\
\text { Dayton, OH }\end{array}$ \\
\hline $10^{*}$ & Erie, Pennsylvania & 42.13 & -80.09 & 2003-2007, 2009-2010 & $\begin{array}{l}\text { Philip E. Gallagher, MD FAAAAI } \\
\text { Allergy \& Asthma Associates of Northeastern PA } \\
\text { Erie, PA }\end{array}$ \\
\hline 11 & Hamilton, Ontario & 43.25 & -79.1 & $2003-2005$ & $\begin{array}{l}\text { Jason A. Ohayan, MD } \\
\text { Hamilton, ON }\end{array}$ \\
\hline 12 & Indianapolis, IN & 39.78 & -86.15 & $2003-2009$ & $\begin{array}{l}\text { L.Y. Frank Wu, M.D. } \\
\text { St. Vincent Professor Building } \\
\text { Indianapolis, IN }\end{array}$ \\
\hline 13 & Kansas City, MO & 39.12 & -94.55 & 2003-2007, 2009-2010 & $\begin{array}{l}\text { Jay Portnoy, MD FAAAAI } \\
\text { Children's Mercy Hospital } \\
\text { Kansas City, MO }\end{array}$ \\
\hline 14 & La Crosse, WI & 43.83 & -91.23 & $2003-2010$ & $\mathrm{~N} / \mathrm{A}$ \\
\hline
\end{tabular}




\begin{tabular}{|c|c|c|c|c|c|}
\hline 15 & Lexington, $\mathrm{KY}$ & $\begin{array}{l}38.04 \\
3\end{array}$ & -84.46 & 2003-2006,2008-2010 & $\begin{array}{l}\text { Beth Miller, M.D. } \\
\text { University of Kentucky Asthma Allergy \& } \\
\text { Immunology }\end{array}$ \\
\hline 16 & Lincoln, NE & 40.82 & -96.69 & $2004-2009$ & $\begin{array}{l}\text { Fred Keichel, MD } \\
\text { Allergy, Asthma \& Immunology Associates } \\
\text { Lincoln, NE }\end{array}$ \\
\hline 17 & London, ON & 42.98 & -81.25 & $2003-2005$ & $\begin{array}{l}\text { James Anderson, MLT } \\
\text { OSHTECH } \\
\text { London, ON }\end{array}$ \\
\hline 18 & Louisville, KY & 38.22 & -85.74 & $2003-2010$ & $\begin{array}{l}\text { James L. Sublett, MD FAAAAI } \\
\text { Family Allergy \& Asthma } \\
\text { Louisville, KY }\end{array}$ \\
\hline 19 & Madison, WI & 43.08 & -89.39 & $2003-2010$ & $\begin{array}{l}\text { Robert Bush, MD FAAAAI } \\
\text { UW Medical School } \\
\text { Madison, WI }\end{array}$ \\
\hline 20 & Melrose Park, IL & 41.90 & -87.86 & $2003-2010$ & $\begin{array}{l}\text { Joseph G. Leija, MD FAAAAI } \\
\text { Dr. Joseph Leija } \\
\text { Melrose Park, IL }\end{array}$ \\
\hline 21 & Minneapolis, MN & 44.96 & -93.27 & 2010 & $\begin{array}{l}\text { Harold B. Kaiser, MD FAAAAI } \\
\text { Clinical Research Institute } \\
\text { Minneapolis, MN }\end{array}$ \\
\hline 22 & Newark, NJ & 40.72 & -74.17 & $2003-2009$ & $\begin{array}{l}\text { Alan Wolff, M.D. } \\
\text { UMDNJ } \\
\text { Newark, NJ }\end{array}$ \\
\hline
\end{tabular}




\begin{tabular}{|c|c|c|c|c|c|}
\hline $23^{*}$ & New Castle, DE & 39.62 & -75.56 & $2005-2010$ & $\begin{array}{l}\text { Michael McDowell } \\
\text { Division of Air Quality, DNREC, State of Delaware } \\
\text { New Castle, DE }\end{array}$ \\
\hline 24 & New York, NY & 40.08 & -78.43 & $2003-2010$ & $\begin{array}{l}\text { Guy Robinson, PhD } \\
\text { Fordham College at Lincoln Center } \\
\text { New York, NY }\end{array}$ \\
\hline 25 & Niagrara Falls, ON & 43.09 & -79.09 & $2003-2005$ & $\begin{array}{l}\text { Dr. Michael Alexander, MD } \\
\text { Niagara Falls, ON }\end{array}$ \\
\hline 26 & Olean, NY & 42.08 & -78.43 & $2003-2010$ & $\begin{array}{l}\text { Fred Lewis, MD FAAAAI } \\
\text { Olean, NY }\end{array}$ \\
\hline 27 & Philadelphia, PA & 40.01 & -75.13 & $2003-2007,2010$ & $\begin{array}{l}\text { Donald J. Dvorin, MD FAAAAI } \\
\text { Allergic Disease Associates, P.C. } \\
\text { Philadelphia, PA }\end{array}$ \\
\hline 28 & Pittsburgh, PA (2) & 40.44 & -79.98 & $2003-2010$ & $\begin{array}{l}\text { David Skoner, MD FAAAAI } \\
\text { Allegheny General Hospital } \\
\text { Pittsburgh, PA } \\
\text { David Nash, M.D. } \\
\text { Children's Hosp. of Pittsburgh }\end{array}$ \\
\hline 29 & Rochester, NY & 43.17 & -77.62 & $2003-2010$ & $\begin{array}{l}\text { Donald W. Pulver, MD FAAAAI } \\
\text { Allergy, Asthma \& Immunology of Rochester } \\
\text { Rochester, NY }\end{array}$ \\
\hline
\end{tabular}




\begin{tabular}{|c|c|c|c|c|c|}
\hline 30 & Salem, MA & 42.53 & -70.87 & $2007-2010$ & $\begin{array}{l}\text { Paul Hannaway, M.D. } \\
\text { Salem, MA }\end{array}$ \\
\hline 31 & St. Claire Shores, MI & 42.49 & -82.89 & 2003-2005, 2009-2010 & $\begin{array}{l}\text { Andrew I. Dzul, MD } \\
\text { Lakeshore Ear Nose \& Throat Center } \\
\text { St. Clair Shores, MI }\end{array}$ \\
\hline 32 & St. Louis, MO & 38.64 & -90.24 & $2003,2006-2010$ & $\begin{array}{l}\text { Mr. Wayne Wilhelm } \\
\text { St. Louis County Health Department } \\
\text { Berkeley, MO (St. Louis) }\end{array}$ \\
\hline 33 & Washington, DC & 38.91 & -77.02 & $2003-2010$ & $\begin{array}{l}\text { Susan E. Kosisky MA } \\
\text { Walter Reed Army Medical Ctr. } \\
\text { Washington, DC }\end{array}$ \\
\hline 34 & Waturbury, CT & 41.56 & -73.04 & $2003-2010$ & $\begin{array}{l}\text { Christopher Randolph, MD FAAAAI } \\
\text { Waterbury, CT }\end{array}$ \\
\hline 35 & Waukesha, WI & 43.01 & -88.24 & $2007-2009$ & $\begin{array}{l}\text { Walter Brummund, MD, PhD, FAAAAI } \\
\text { Allergy \& Asthma Centers, S.C. } \\
\text { Waukesha, WI }\end{array}$ \\
\hline 36 & Wauwatosa, WI & 43.06 & -88.03 & $2003-2006$ & $\begin{array}{l}\text { Walter Brummund, MD, PhD, FAAAAI } \\
\text { Allergy \& Asthma Centers, S.C. } \\
\text { Waukesha, WI }\end{array}$ \\
\hline 37 & York, PA & 39.96 & -76.73 & $2003-2010$ & $\begin{array}{l}\text { Michael S. Nickels, MD PhD } \\
\text { Allergy and Asthma Consultants, Inc. } \\
\text { York, PA }\end{array}$ \\
\hline
\end{tabular}




\begin{tabular}{|c|c|c|c|c|c|}
\hline & ast (33) & & & & \\
\hline 1 & Atlanta, GA & 33.76 & -84.42 & $2003-2010$ & $\begin{array}{l}\text { Santley M. Fineman, MD MBA FAAAAI } \\
\text { Atanta Allergy and Asthma } \\
\text { Marietta, GA (Atlanta) }\end{array}$ \\
\hline 2 & Austin, TX & 30.31 & -97.95 & $2003-2005$ & $\begin{array}{l}\text { Kim T. Hovanky, MD FAAAAI \& } \\
\text { Sheila M. Amar, MD FAAAAI, FACAAI } \\
\text { Allergy \& Asthma Center of Georgetown }\end{array}$ \\
\hline 3 & Baton Rouge, Louisiana & 30.45 & -91.13 & $2003-2005$ & $\begin{array}{l}\text { James M. Kidd III } \\
\text { Kidd Allergy Clinic } \\
\text { Baton Rouge, LA }\end{array}$ \\
\hline 4 & Birmingham, AL & 33.53 & -86.80 & 2010 & $\begin{array}{l}\text { Weilly Soong, MD FAAAAI } \\
\text { Birmingham-Southern College/Alabama Allergy \& } \\
\text { Asthma Center }\end{array}$ \\
\hline 5 & Charlotte, $\mathrm{NC}$ & 35.20 & -80.83 & $2003-2007$ & $\begin{array}{l}\text { John T. Klimas, MD FAAAAI } \\
\text { Carolina Asthma and Allergy Center } \\
\text { Charlotte, NC }\end{array}$ \\
\hline 6 & College Station, TX & 30.60 & -96.31 & $2003-2010$ & $\begin{array}{l}\text { David R. Weldon, MD FAAAAI, FACAAI } \\
\text { Scott \& White Clinic } \\
\text { College Station, TX }\end{array}$ \\
\hline 7 & Corpus Christi, TX & 27.69 & -97.29 & $2003-2005$ & $\begin{array}{l}\text { Gary L. Smith, M.D. } \\
\text { Corpus Christi, TX }\end{array}$ \\
\hline
\end{tabular}




\begin{tabular}{|c|c|c|c|c|c|}
\hline 8 & Dallas, TX & 32.79 & -96.77 & $2003-2010$ & $\begin{array}{l}\text { Jeffrey Adelglass, M.D. } \\
\text { Dr. Jeffrey Adelglass } \\
\text { Dallas, TX }\end{array}$ \\
\hline 9 & Fargo, ND & 46.88 & -96.82 & $2003-2010$ & $\begin{array}{l}\text { Dan Dalan, MD FAAAAI } \\
\text { Allergy \& Asthma Care Center } \\
\text { Fargo, ND }\end{array}$ \\
\hline 10 & Flower Mound, TX & 33.03 & -97.09 & $2006-2010$ & $\begin{array}{l}\text { Marie H Fitzgerald, MD } \\
\text { North Texas Pollen Station } \\
\text { Flower Mound, TX }\end{array}$ \\
\hline 11 & Fort Smith, AK & 35.37 & -94.38 & $2003-2006$ & N/A \\
\hline 12 & Georgetown, TX & 30.65 & -97.69 & $2006-2010$ & $\begin{array}{l}\text { Kim T. Hovanky, MD FAAAAI \& } \\
\text { Sheila M. Amar, MD FAAAAI, FACAAI } \\
\text { Allergy \& Asthma Center of Georgetown }\end{array}$ \\
\hline 13 & Greenville, SC & 34.84 & -82.37 & $2003-2010$ & $\begin{array}{l}\text { Neil L Kao MD FAAAAI } \\
\text { Allergic Disease and Asthma Center } \\
\text { Greenville, SC }\end{array}$ \\
\hline 14 & Houston, TX & 29.77 & -95.39 & $2005-2010$ & $\begin{array}{l}\text { Mr. Tony Huynh } \\
\text { City of Houston } \\
\text { Houston, TX }\end{array}$ \\
\hline 15 & Huntsville, AL & 34.71 & -86.63 & $2003-2010$ & $\begin{array}{l}\text { Ms. Debra Hopson } \\
\text { Natural Resources \& Environmental Management } \\
\text { Huntsville, AL }\end{array}$ \\
\hline
\end{tabular}




\begin{tabular}{|c|c|c|c|c|c|}
\hline 16 & Knoxville, TN & 35.97 & -83.95 & $2003-2010$ & $\begin{array}{l}\text { Michael Miller, MD FAAAAI } \\
\text { Allergy, Asthma and Immunology } \\
\text { Knoxville, TN }\end{array}$ \\
\hline 17 & Little Rock, AK & 34.72 & -92.35 & $2004-2010$ & $\begin{array}{l}\text { Karl V Sitz, MD } \\
\text { Little Rock Allergy \& Asthma Clinic } \\
\text { Little Rock, AR }\end{array}$ \\
\hline 18 & Miami, FL & 25.78 & -80.21 & $2003-2005$ & $\begin{array}{l}\text { Elene Ubals, MD \& } \\
\text { Richard Schiff, MD, PhD } \\
\text { Miami, FL }\end{array}$ \\
\hline 19 & New Orleans, LA & 30.07 & -89.93 & $2008-1010$ & $\begin{array}{l}\text { W Edward Davis MD MS MBA MMM } \\
\text { Ochsner Clinic Foundation } \\
\text { New Orleans, LA }\end{array}$ \\
\hline 20 & Ocala, FL & 29.19 & -82.13 & $2003-2005$ & $\begin{array}{l}\text { Dr. Karl M Altenburger } \\
\text { Allergy and Asthma Care of Florida } \\
\text { Ocala, FL }\end{array}$ \\
\hline 21 & Oklahoma City, OK (2) & 35.47 & -97.51 & $2003-2010$ & $\begin{array}{l}\text { Warren V. Filley, MD FAAAAI } \\
\text { OK Allergy Asthma Clinic, Inc. } \\
\text { Oklahoma City, OK } \\
\text { Martha Tarpay, MD } \\
\text { Allergy \& Asthma Center }\end{array}$ \\
\hline 22 & Omaha, NE & 41.26 & -96.01 & $2003-2010$ & N/A \\
\hline
\end{tabular}




\begin{tabular}{|c|c|c|c|c|c|}
\hline 23 & Orlando, FL & 28.51 & -81.37 & $2006-2007$ & $\begin{array}{l}\text { Bruce A. Hornberger, MD FAAAAI } \\
\text { Allergy \& Asthma Center of East Orlando } \\
\text { Orlando, FL }\end{array}$ \\
\hline 24 & Oxford, AL & 33.61 & -85.84 & 2010 & $\begin{array}{l}\text { Robert Grubbe, MD } \\
\text { Allergy \& Asthma Center, LLC } \\
\text { Oxford, AL }\end{array}$ \\
\hline 25 & Rogers, AK & 36.33 & -94.13 & $2006-2010$ & $\begin{array}{l}\text { Curtis L. Hedberg, MD FAAAAI } \\
\text { Hedberg Allergy \& Asthma Center } \\
\text { Rogers, AR (Fort Smith) }\end{array}$ \\
\hline $26^{*}$ & Sarasota, FL & 27.34 & -82.53 & $2003-2010$ & $\begin{array}{l}\text { Mary Jelks, MD FAAAAI } \\
\text { Sarasota, FL }\end{array}$ \\
\hline 27 & Savannah, GA & 32.02 & -81.13 & $2008-2010$ & $\begin{array}{l}\text { Brad H. Goodman, M.D. \& } \\
\text { Bruce D. Finkel, M.D. } \\
\text { Coastal Allergy \& Asthma, P.C. }\end{array}$ \\
\hline 28 & Tallahasseee, FL & 30.46 & -84.28 & $2003-2005$ & $\begin{array}{l}\text { Ronald Saff, M.D. } \\
\text { Tallahassee, FL }\end{array}$ \\
\hline 29 & Tampa, FL & 27.96 & -82.48 & $2003-2010$ & $\begin{array}{l}\text { Richard Lockey, MD FAAAAI } \\
\text { University of South Florida } \\
\text { Tampa, FL }\end{array}$ \\
\hline 30 & Tulsa, OK & 36.13 & -95.92 & $2003-2010$ & $\begin{array}{l}\text { Estelle Levetin, PhD FAAAAI } \\
\text { University of Tulsa } \\
\text { Tulsa, OK }\end{array}$ \\
\hline
\end{tabular}




\begin{tabular}{|c|c|c|c|c|c|}
\hline 31 & Waco, TX (2) & 31.57 & -97.18 & $2003-2010$ & $\begin{array}{l}\text { N.J. Amar, MD FAAAAI } \\
\text { Allergy and Asthma Center } \\
\text { Waco, TX } \\
\text { Pramila K. Daftary, MD FAAAAI }\end{array}$ \\
\hline \multicolumn{6}{|c|}{ Mountain (8) } \\
\hline 1 & Bismarck, ND & 46.81 & -100.77 & $2003-2005,2010$ & $\begin{array}{l}\text { Dan Dalan, M.D. } \\
\text { North Dakota DOH East Lab } \\
\text { Bismarck, ND }\end{array}$ \\
\hline 2 & Colorado Springs (2) & 38.86 & -104.76 & $2003-2010$ & $\begin{array}{l}\text { William Storms, MD FAAAAI } \\
\text { The William Storms Allergy Clinic } \\
\text { Colorado Springs, CO } \\
\text { Robert A. Nathan, MD FAAAAI \& Daniel F. Soteres, } \\
\text { MD MPH FAAAAI }\end{array}$ \\
\hline 3 & Draper, UT & 40.52 & -111.86 & $2009-2010$ & $\begin{array}{l}\text { Duane J. Harris, MD FAAAAI } \\
\text { Intermountain Allergy \& Asthma Clinic } \\
\text { Draper, UT }\end{array}$ \\
\hline $4 *$ & Missoula, MT & 46.87 & -114.01 & 2006-2007, 2009-2010 & $\begin{array}{l}\text { Emily Weiler } \\
\text { U of Montana, Ctr for Environmental Health Sciences } \\
\text { Missoula, MT }\end{array}$ \\
\hline
\end{tabular}




\begin{tabular}{|l|l|l|l|l|l|}
\hline 5 & Salt Lake City, UT & 40.78 & -111.93 & $2003-2004,2008$ & N/A \\
\hline 6 & Scottsdale, AZ & 33.69 & -111.87 & $2006-2007$ & $\begin{array}{l}\text { Michael E. Manning, M.D. } \\
\text { Scottsdale, AZ }\end{array}$ \\
\hline $7^{*}$ & Twin Falls, ID & 42.56 & -114.46 & $2003-2010$ & N/A \\
\hline
\end{tabular}

California (13)

\begin{tabular}{|l|l|l|l|l|l|}
\hline 1 & La Jolla, CA & 32.84 & -117.26 & $2003-2010$ & $\begin{array}{l}\text { Robert Reid, Jr, MD } \\
\text { Scripps Memorial Hospital } \\
\text { LaJolla, CA }\end{array}$ \\
\hline 2 & Orange, CA & 33.81 & -117.82 & $2003-2010$ & $\begin{array}{l}\text { Sherwin A. Gillman, M.D. } \\
\text { Children's Hospital of Orange County-Pediatric } \\
\text { Subspecialty Faculty } \\
\text { Puilip Taylor, PhD } \\
\text { The Pollen Group } \\
\text { Pasadena, CA }\end{array}$ \\
\hline 3 & Pasadena, CA & 34.16 & -118.14 & $2004-2005,2010$ & N/A \\
\hline 4 & Pleasanton, CA & 37.67 & -121.89 & $2003-2010$ & \\
\hline
\end{tabular}




\begin{tabular}{|c|c|c|c|c|c|}
\hline 5 & Reno, NV & 39.54 & -119.82 & 2003-2006, 2009-2010 & $\begin{array}{l}\text { Leonard Shapiro, MD FAAAAI } \\
\text { Allergy \& Asthma Associates } \\
\text { Sparks, NV }\end{array}$ \\
\hline 6 & Roseville, CA & 38.76 & -121.29 & $2006-2010$ & $\begin{array}{l}\text { Sunil P. Perera, MD FAAAAI } \\
\text { Allergy Medical Group of the North Area } \\
\text { Roseville, CA (Sacremento) }\end{array}$ \\
\hline 7 & Sacramento, CA & 38.57 & -121.47 & $2003-2006$ & $\begin{array}{l}\text { Sunil P. Perera, MD FAAAAI } \\
\text { Allergy Medical Group of the North Area } \\
\text { Roseville, CA (Sacremento) }\end{array}$ \\
\hline 8 & Salinas, CA & 36.68 & -121.64 & 2003 & $\begin{array}{l}\text { Steven S. Prager, M.D. } \\
\text { Salinas Allergy Clinic } \\
\text { Salinas, CA }\end{array}$ \\
\hline 9 & San Diego, CA & 32.82 & -117.14 & $2006,2008-2010$ & $\begin{array}{l}\text { Robert T. Reid, MD } \\
\text { Erik and Ese Banck Clinical Research Center } \\
\text { San Diego, CA }\end{array}$ \\
\hline 10 & San Jose, CA (2) & 37.30 & -121.85 & $2003-2010$ & $\begin{array}{l}\text { Theodore Chu, MD FAAAAI } \\
\text { San Jose, CA }\end{array}$ \\
\hline 11 & Santa Barbara, CA & 34.43 & -119.72 & $2003-2010$ & $\begin{array}{l}\text { Myron Liebhaber, M.D. } \\
\text { Sansum - Santa Barbara Medical Foundation Clinic } \\
\text { Santa Barbara, CA }\end{array}$ \\
\hline 12 & Stockton, CA & 37.97 & -121.31 & $2009-2010$ & $\begin{array}{l}\text { Gregory W. Bensch, MD FAAAAI \& George W } \\
\text { Bensch, MD FAAAAI } \\
\text { Allergy, Immunology and Asthma Medical Group }\end{array}$ \\
\hline
\end{tabular}




\begin{tabular}{|c|c|c|c|c|c|}
\hline \multicolumn{6}{|c|}{ Pacific NW (4) } \\
\hline 1 & Crescent City, CA & 41.76 & -124.20 & $2009-2010$ & $\mathrm{~N} / \mathrm{A}$ \\
\hline $2 *$ & Eugene, OR & 44.05 & -123.11 & $2003-2010$ & $\begin{array}{l}\text { Kraig W. Jacobson, MD, FAAAAI } \\
\text { Allergy \& Asthma Research Group } \\
\text { Eugene, OR }\end{array}$ \\
\hline 3 & Seattle, WA & 47.62 & -122.35 & $2003-2010$ & $\mathrm{~N} / \mathrm{A}$ \\
\hline $4 *$ & Vancouver, WA & 45.63 & -122.64 & $2003-2010$ & $\begin{array}{l}\text { Raymond Brady, M.D. and Joseph Hassett, M.D. } \\
\text { Vancouver, WA }\end{array}$ \\
\hline
\end{tabular}

Table S1. AAAAI pollen counting stations included in development and evaluation of model. Categorized by U.S. subregions Northeast, Southeast,

805 Mountain, California, and Pacific Northwest. Sites equipped with two samplers are denoted in parentheses (ex. Pittsburgh, PA (2)). Station numbers with asterisk (*) are collocated with DayMet temperature for phenological regressions. 


\begin{tabular}{|c|c|c|c|}
\hline Taxon & 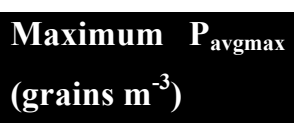 & 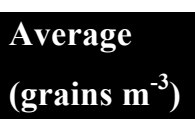 & $\mathbf{P}_{\text {avgmax }}$ \\
\hline Acer & 535 & 212 & \\
\hline Alnus & 176 & 73 & \\
\hline Ambrosia & 379 & 193 & \\
\hline Arecaceae & 27 & 13 & \\
\hline Artemisia & 76 & 36 & \\
\hline Asteraceae & 64 & 28 & \\
\hline Betula & 564 & 232 & \\
\hline Carpinus/Ostrya & 71 & 29 & \\
\hline Carya & 126 & 54 & \\
\hline Celtis & 155 & 65 & \\
\hline Chenopodiaceae/Amaranthaceae & 80 & 29 & \\
\hline Corylus & 13 & 5 & \\
\hline Cupressaceae & 1949 & 960 & \\
\hline Cyperaceae & 37 & 12 & \\
\hline Eupatorium & 27 & 13 & \\
\hline Fagus & 66 & 17 & \\
\hline Fraxinus & 462 & 227 & \\
\hline Graminae / Poaceae & 197 & 98 & \\
\hline Juglans & 71 & 34 & \\
\hline Ligustrum & 13 & 6 & \\
\hline Liquidambar & 109 & 44 & \\
\hline Morus & 940 & 398 & \\
\hline Myrica & 50 & 25 & \\
\hline Olea & 197 & 43 & \\
\hline Other Grass Pollen & 85 & 42 & \\
\hline Other Tree Pollen & 863 & 332 & \\
\hline Other Weed Pollen & 160 & 51 & \\
\hline Pinaceae & 645 & 328 & \\
\hline Plantago & 33 & 13 & \\
\hline Platanus & 191 & 103 & \\
\hline Populus & 367 & 158 & \\
\hline Prosopis & 34 & 16 & \\
\hline Pseudotsuga & 7 & 3 & \\
\hline
\end{tabular}




\begin{tabular}{|l|l|l|}
\hline Quercus & 2106 & 1081 \\
\hline Rumex & 59 & 23 \\
\hline Salix & 123 & 58 \\
\hline Tilia & 19 & 6 \\
\hline Tsuga & 12 & 5 \\
\hline Typha & 19 & 10 \\
\hline Ulmus & 556 & 249 \\
\hline Unidentified Pollen & 198 & 57 \\
\hline Urticaceae & 86 & 36 \\
\hline
\end{tabular}

809 SI Table 2. Criteria scores used for selecting pollen taxa for this study. Shaded taxa are selected taxa based on

810 minimum of 1) 100 grains $\mathbf{m}^{-3}$ for the maximum $\boldsymbol{P}_{\text {avgmax }}$ and 2) $\mathbf{7 0}$ grains $\mathbf{m}^{-3}$ for the maximum $\boldsymbol{P}_{\text {avgmax }}$.

811

812 


\section{program PECM}

\section{! MODEL VERSION: PECM1.0}

! Final Code Update: Matthew Wozniak, 08/02/16

! Last ALS update: 1 June 2015 - substantial updates to data input; creation of netcdf output, phenological calculations

! Based on earlier version (beldread.f90, partially developed by MAZ)

! UPDATE: now includes normalization of emission factor (normalized to Gaussian curve) $(06 / 2015)$

! UPDATE: phenology now parameterized with sDOY and eDOY versus PYAAT (5/2016)

! UPDATE: final emissions output is aggregated by PFT (DBL, ENL, Grasses, Ragweed)

! UPDATE: Ragweed landcover is given by CLM crop (corn and soybean) PFTs and CLM urban level 3. Parameterization is drawn from Clay et al. 2006 (crop ragweed) and Katz et al. 2014 (urban ragweed) ragweed densities.

! Crops are split into tilled vs. no-till (fractions given by USDA)

! UPDATE: Ragweed crop parameterization reduced by a factor of 100 based on comparison of test-run RegCM-pollen simulated surface counts versus observed ragweed counts. Grass

! UPDATE: Grasses' emission factor has been reduced to minimum from literature for $\mathrm{C} 3$ and $\times 10^{\wedge}-1$ the minimum for $\mathrm{C} 4$

\section{use netcdf}

\section{implicit none}

logical :: plac_flag

integer : :

nx , ny , nyr, ndy , ndy_nextyr, nspec, nbeld, nclm, cnt, cnt_yrs, cnt_mnths, nlev, yrst, yrend, ndytot, ngra, nrag ! mcwoz added nlev, yrst, yrend, ndytot, nclm, ngra, nrag

integer :: idbeld, idLat, idLong, idyear, idday, idclm, idpft, idurb, idc3, idc4, idurblev ! mcwoz added idc3, idc4, idurblev

integer :: idcru, idTmp, status, dimval, nlats

integer :: len, wid

integer :: phen_dimids(4), phen_count (4)

integer : : i, ii, iii, iv, iv2,jj,id_2d(2), start(4), count(4), start1(4), count1(4), rcode, pcoutid, ncidout ! mcwoz added $j j$

integer :: dimid1, dimid2,dimid3, dimid4,dimid5, dimids(4), dimid6, dimid7, 1onoutid, latoutid, dayoutid, yroutid, specoutid, timeoutid, levoutid, lcoutid, sdoyoutid, edoyoutid, duroutid, dbloutid, enloutid,graoutid, ragoutid ! mcwoz added dbl-, enl-, gra-, ragoutid

real,allocatable : : sDOY_m(:), sDOY_b(:), eDOY_m(: ), eDOY_b(: ), ef(: )

double precision, allocatable $:: \operatorname{tval}(:,:,: \overline{)}, \operatorname{pft}(:,:, \bar{l}), \operatorname{urban}(:,:,:)$ real, allocatable : : $x \operatorname{lat}(:,:), \operatorname{xlon}(:,:), \operatorname{grass}(:,:)$

integer, allocatable $::$ ispec $(:)$, idspec $(:), \operatorname{ttest}(:,:,:)$, lattest $(:,:)$

integer, allocatable $:$ : iyr(:),idy(:),idcrop(:) ! mcwoz added idcrop

real, allocatable $::$ pyaat_sum $(:,:,:)$, pyaat $(:,:,:)$, pyaat_sum_adj $(:,:,:)$,

pyaat_adj $(:,:,:)$ ! pyat $=$ prior year annual average temperature

real, allocatable : : frac_area $(:,:,:), \operatorname{beld}(:,:,:), \operatorname{sDoY}(:,:,:,:), \operatorname{edoY}(:,:,:,:)$, $\operatorname{dur}(:,:,:,:), \operatorname{pc}(:,:,:,:), \mathrm{dbl} \_p o l l(:,:,:,:)$, enl_poll $(:,:,:,:), \operatorname{gra}$ _poll $(:,:,:$ $,:)$, rag_poll $(:,:,:,:)$

real, dimension $(103,2)::$ stat_llind

real : : sigma, mu, gphen,sigma1,sigma2,mu1,mu2,tot_flx,ef_d! mcwoz added tot_flx, ef_d 
real : : vf $=0.10$ ! for application of flowering probability (vitality) to emission factor

character(len=256) :: longname(8), units(8), lc_option(1)

character(4), allocatable :: name(:), year(:)

yrst $=1$ ! start year of emissions

yrend $=11$ ! end year of emissions

nyr $=$ yrend-yrst +1

units $(4)=$ 'days since 2000-12-31 00:00:00'

! Indicate below which land cover specification to use ("PFT" = PFT-level from CLM, "TAX" taxon-level from BELD)

lc_option = "PFT"

if (lc_option(1:3) .eq. "TAX") then

! Read input file scalars

open ( unit=20, file=' emission_params_beld.in ' , status= ' unknown' )

$\operatorname{read}(20, *) \mathrm{nx}$ (longitude)

$\operatorname{read}(20, *)$ ny

( latitude)

read $(20, *)$ ndy definition)

read $(20, *)$ nbeld database (currently 11)

read $(20, *)$ ngra C4)

read $(20, *)$ nrag

$\operatorname{read}(20, *)$ idc 3

species data

$\operatorname{read}(20, *)$ idc 4

read $(20, *)$ idurblev

parameterization

read $(20, *)$ nspec model

read $(20, *)$ nlev

! Number of grid points in $\mathrm{x}$ direction

! Number of grid points in y direction

! number of days per year (calendar

! number of tree taxa in the BELD species

! number of grass taxa (currently 2: C3 and

! number of ragweed taxa (currently 1)

! index of c3 non-arctic grasses pft in CLM

! index of c4 grasses pft in CLM species data

! index of the urban level used for ragweed

! number of pollen genus/type to include in

! number of vertical levels in emissions model

allocate ( year(nyr))

year $=$

(/'2001', '2002', '2003', '2004', '2005', '2006' , '2007' , '2008' , '2009' , '2010', ' 20 $\left.11^{\prime} /\right)$

! count the total number of days in the emissions calculation accounting for leap days

ndytot $=0$

do iv = yrst, yrend

if (year(iv) .eq. '2004' .or. year(iv) .eq. '2008') then

else ndytot $=$ ndytot +366

end if

ndytot $=$ ndytot +365

end do

!--- Allocate memory for arrays

allocate $(x \operatorname{lon}(n x, n y))$ ! note CRU dims are different

allocate $(x \operatorname{lat}(n x, n y))$

allocate(iyr(nyr)) 


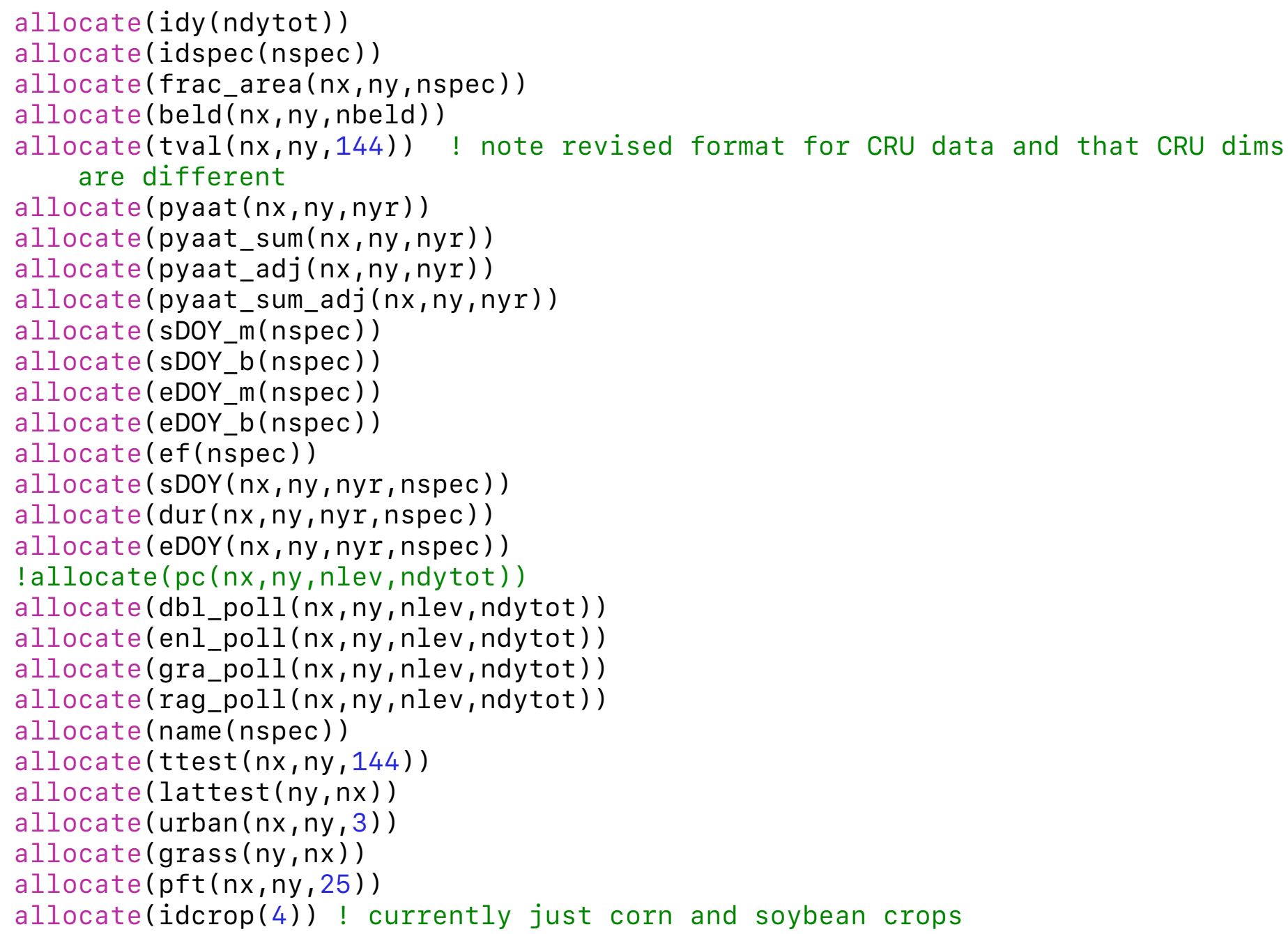

! print the phenological parameters to screen

do $i=1$, nspec

write $(*, *)$ name $(i)$,ef(i), sDOY_m(i), sDOY_b(i) ,eDOY_m(i), eDOY_b(i)

end do 
!---Read in BELD3 $25 \mathrm{~km}$ res lat/lon file-

status $=$ nf90_open (path $=$ "/good_share/alsteine/pollendata/

merged_beld_25km.nc", mode = nf90_nowrite, ncid = idbeld)

do $i=1$, nbeld

status = nf90_inq_varid(idbeld, name(i), idspec(i))

status $=$ nf90_get_var $(i d b e l d, i d \operatorname{spec}(i), \operatorname{beld}(:,:, i))$

end do

frac_area $(:,:, 1: \operatorname{nbeld})=\operatorname{beld}(:,:,:)$

status $=$ nf90_close $($ idbeld $)$

! read in CLM land cover data (PFTS for grasses and crops, urban landcover)

status $=$ nf90_open $($ path $=$ "/home/mcwoz/CLM45_surface_emisvars.nc", mode $=$ nf90 nowrite, ncid = idclm)

status = nf90_inq_varid(idclm, 'pft_2d', idpft)

status $=$ nf90_get_var $(i d c l m$, idpft, pft $)$

status = nf90_inq_varid(idclm, 'urb_2d', idurb)

status = nf90_get_var(idclm, idurb, urban $)$

! Duplicate the elm landcover

! do $i=1$, ny

! do $i \mathrm{i}=1, \mathrm{nx}$

! frac_area $(i i, i, 12)=$ frac_area $(i i, i, 11)$

! end do

! end do

frac_area $(:,:, 12)=$ frac_area $(:,:, 11)$

frac_area $(:,:)=,0.01 * f r a c \_a r e a(:,:$,$) ! convert from percent to fraction (\mathrm{m} 2$ $\mathrm{veg} / \mathrm{m} 2$ total)

$\operatorname{pft}(:,:,:)=0.01 * \operatorname{pft}(:,:,:)$

$\operatorname{urban}(:,:,:)=0.01 * \operatorname{urban}(:,:,:)$

! grass landcover

$\mathrm{nclm}=$ ngra

frac_area $(:,:, 13)=\operatorname{real}(\operatorname{pft}(:,:$, idc3 $), 4)$

frac_area $(:,:, 14)=\operatorname{real}(\operatorname{pft}(:,:$, idc 4$), 4)$

!frac_area $(:,:, 13)=1$.

!frac_area $(:,:, 14)=1$.

! write $(*, *)$ size $(p f t, 1)$, size $(p f t, 2)$, size $(p f t, 3)$

! write $(*, *)$ idc3, idc4

! Ragweed landcover; NOTE: in plants/m2, NOT fractional area

frac_area $(:,:, 15)=$ real $(0.01 *(2 . * 0.75+10 . * 0.25) *(p f t(:,:$, idcrop $(1))+$ $\overline{p f t}(:,:, i d c r o p(2)))+0.01 *(2 . * 0.55+10 . * 0.45) *(p f t(:,:$, idcrop $(3))+$ $\operatorname{pft}(:,:$, idcrop $(4)))+0.1 * 0.5 * \operatorname{urban}(:,:$, idurblev $), 4)$

! (t_density*till + nt_density*no-till)*(corn) + (t_density*till + nt_density*no-till)*(soybean) + urb_density*urbān

! now with crop density reduced by factor of 100, urban reduced by factor of 10

do $i=1, n x$

do $i i=1$, ny

do $\mathrm{iii}=13,15$

if (frac_area(i,ii,iii).1t.0) then

frac_area $(i, i i, i i)=0$. 
end if

end do

end do

end do

end if ! ends "if ('TAX')" block

if (1c_option(1:3) .eq. "PFT") then

! Read input file scalars

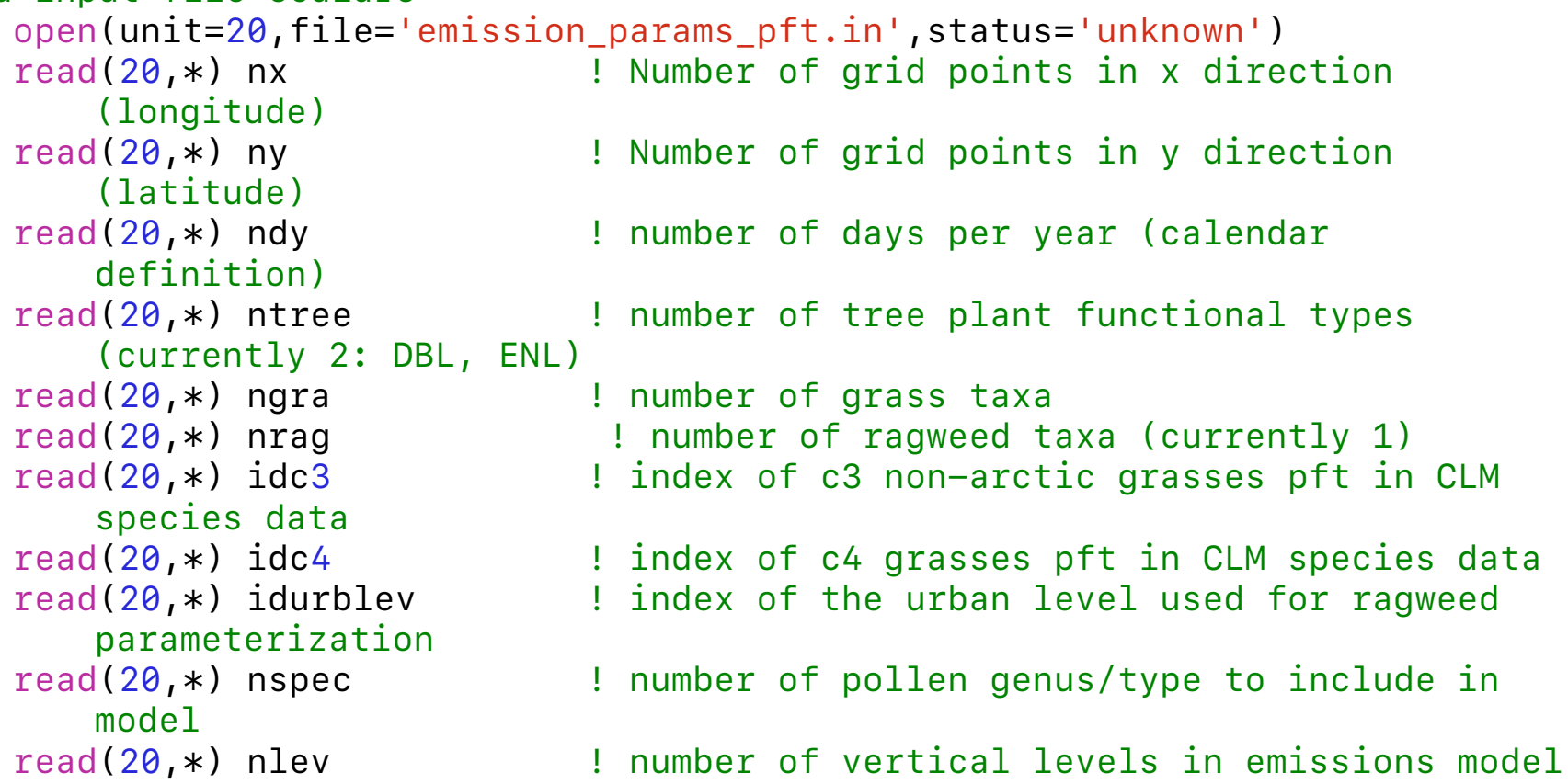

allocate ( year (nyr))

year $=$

(/'2001', '2002', '2003', '2004', '2005', '2006', '2007', '2008' , '2009' , '2010', '20 $\left.11^{\prime} /\right)$

! count the total number of days in the emissions calculation accounting for

leap days

ndytot $=0$

do iv = yrst, yrend

if (year(iv) .eq. '2004' .or. year(iv) .eq. '2008') then ndytot $=$ ndytot +366

else

ndytot $=$ ndytot +365

end if

end do

!--- Allocate memory for arrays

allocate $(x \operatorname{lon}(n x, n y))$ ! note CRU dims are different

allocate (xlat $(n x$, ny $))$

allocate(iyr(nyr))

allocate (idy (ndytot))

allocate (idspec (nspec))

allocate (frac_area $(n x, n y, n s p e c))$

allocate(tval $(\mathrm{nx}, \mathrm{ny}, 144))$ ! note revised format for CRU data and that CRU dims are different 


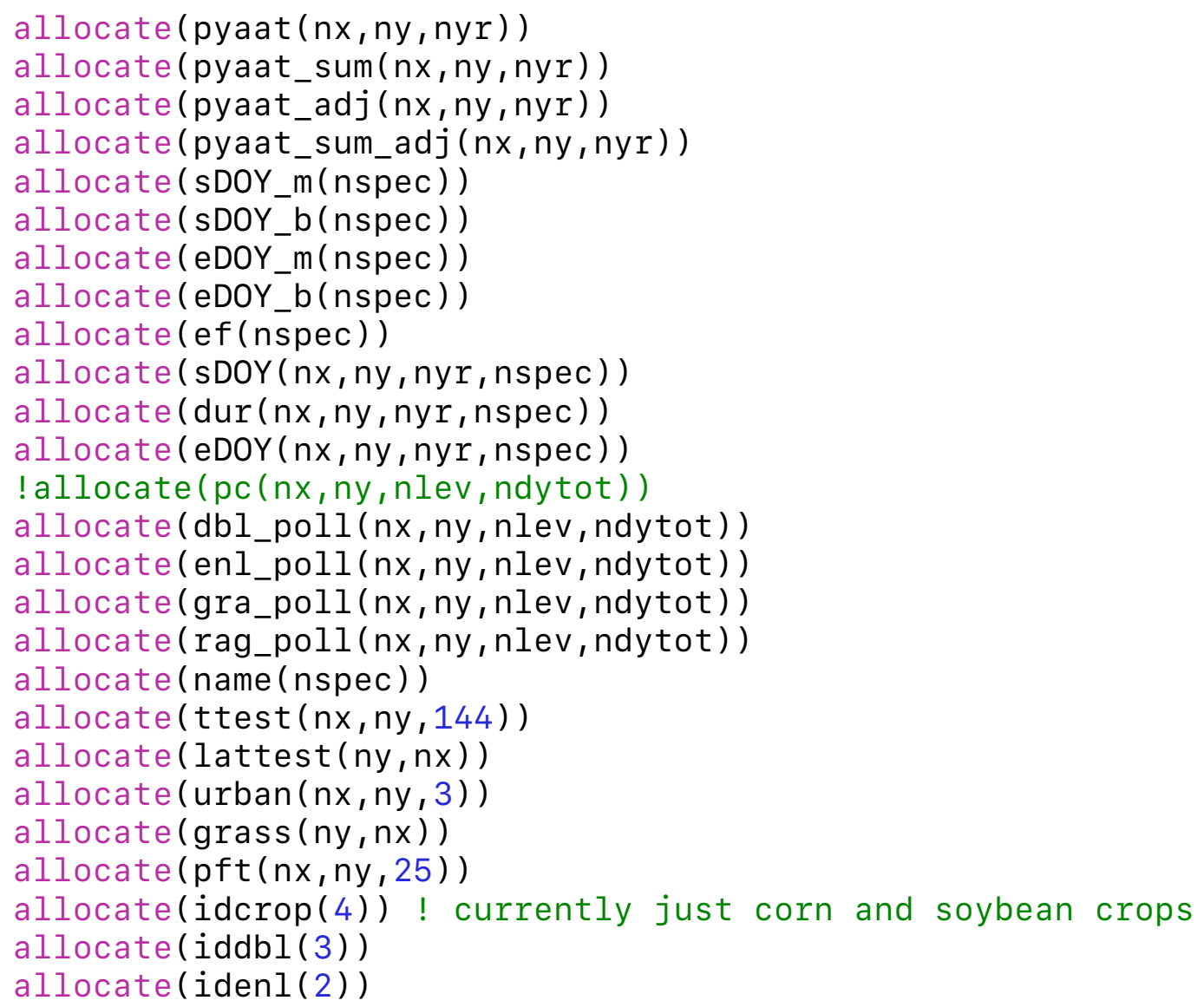

! set idy to integer counting array counting from 1 to the number of days of emissions

idy $=(/(i, i=1$, ndytot $) /)$

iyr $=(/(i, i=1$, nyr $) /)$

! read in rest of input file

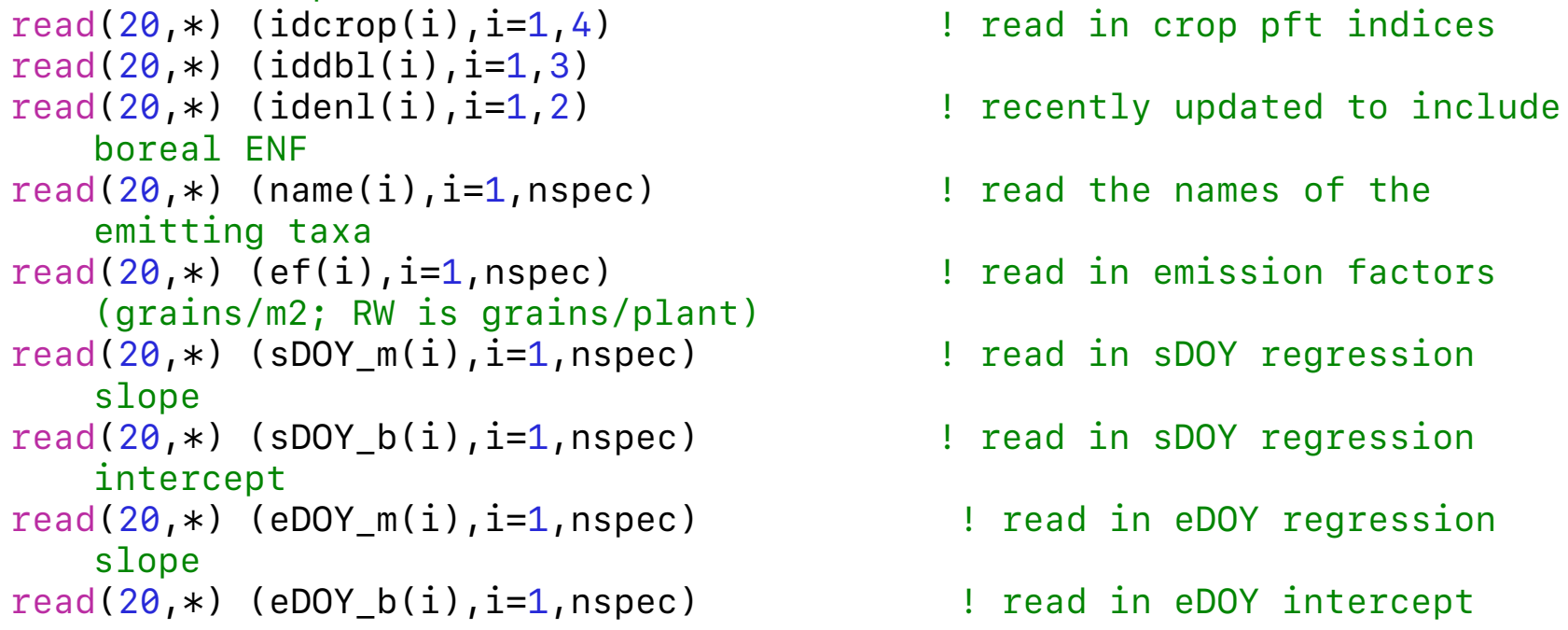

! read in crop pft indices

! recently updated to include

! read the names of the

! read in emission factors

! read in sDOY regression

! read in sDOY regression

! read in eDOY regression

! read in eDOY intercept

! print the phenological parameters to screen

do $i=1$, nspec

write $(*, *)$ name $(i)$,ef $(i), \operatorname{sDOY} m(i), \operatorname{sDOY} \_b(i)$, eDOY_m(i) ,eDOY_b(i)

end do

! read in CLM land cover data (PFTS for grasses and crops, urban landcover) status $=$ nf90_open $($ path $=$ "/home/mcwoz/CLM45_surface_emisvars.nc", mode $=$ nf90 


$$
\text { _nowrite, ncid }=\text { idclm) }
$$

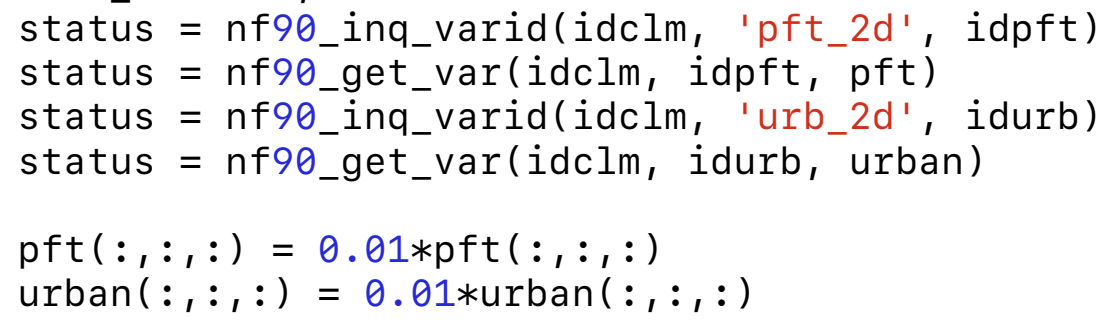

! Ragweed landcover; NOTE: in plants/m2, NOT fractional area

frac_area $(:,:, 5)=\operatorname{real}(0.01 *(2 . * 0.75+10 . * 0.25) *(p f t(:,:$, idcrop $(1))+$ $\operatorname{pft}(:,:$,idcrop (2)) ) $+0.01 *(2 . * 0.55+10 . * 0.45) *(\operatorname{pft}(:,:$, idcrop(3) $)+$ $\operatorname{pft}(:,:$,idcrop $(4)))+0.1 * 0.5 * \operatorname{urban}(:,:$, idurblev $), 4)$

! (t_density*till + nt_density*no-till)*(corn) + (t_density*till + nt_density*no-till)*(soybean) + urb_density*urban

! now with crop density reduced by factor of 100

do $i=1, n x$

do $i i=1$, ny

do $i i i=1,5$

if (frac_area(i,ii,iii).1t.0) then

frac_area $(i, i i, i i i)=0$.

end if

end do

end do

end do

end if ! ends "if ('PFT')" block

! officially close landcover files

status $=$ nf90_close $(i d c l m)$

! officially close landcover files

status $=$ nf90_close $($ idclm $)$

!---Read in CRU daily temperature (Tmax) file-----------------

status = nf90_open $($ path $=$ "/home/mcwoz/cru2rcm_tavg_aat_1999-2010.nc", mode = nf90_nowrite, ncid = idcru)

status = nf90_inq_varid(idcru, "tavg", idTmp)

! 2:nyr because starts with 1999 (predicts 2000), while aatj starts with 1999-2000 (predicts 2000-2001. 
! I want to start with the same prediction year.

status = nf90_get_var(idcru, idTmp, pyaat, $\operatorname{start}=(/ 1,1,2 /)$, count $=(/ \mathrm{nx}$, ny, nyr $/))$

if(status /=nf90_noerr) write $(*, *)$ "netcdf error $=$

$", \operatorname{trim}($ nf90_strerror(status))

!---Read in latitude values

status = nf90_inq_varid(idcru, "xlat", idLat)

status = nf90_get_var(idcru, idLat, $x l$ at, $\operatorname{start}=(/ 1,1 /)$, count $=(/ n x, n y /))$

!status = nf9o_get_var(idcru, idLat, $x$ lat $)$

!---Read in longitude values

status = nf90_inq_varid(idcru, "xlon", idLong)

status = nf90_get_var(idcru, idLong, $x$ lon, $\operatorname{start}=(/ 1,1 /)$, count $=(/ \mathrm{nx}, \mathrm{ny} /))$

! Close the file, freeing all resources.

status $=$ nf90_close $($ idcru $)$

status $=$ nf90_open $($ path $=$ "/home $/ \mathrm{mcwoz} /$ cru2rcm_tavg_aatj_1999-2010.nc", mode $=$ nf90_nowrite, ncid = idcru)

status = nf90_inq_varid(idcru, "tavg", idTmp)

status $=$ nf90_get_var(idcru, idTmp, pyaat_adj, start $=(/ 1,1,1 /)$, count $=(/ n x, n y$, nyr/) )

if(status /=nf90_noerr) write $(*, *)$ "netcdf error $=$

", trim(nf90_strerror(status))

!write $(*, *)$ tval $(101,101,1: 24)$

status $=$ nf90_close $($ idcru $)$

if (.false.) then ! on/off - calculate pyaat from monthly temperature data

!---Calculate prior year annual average temperature (spring taxa; start in January)

pyaat_sum $=-9999$.

pyaat $=-9999$.

do $i=1, n x \quad$ ! Loop over all longitudes

do $\mathrm{ii}=1$, ny ! Loop over all latitudes

cnt_yrs = 1 ! reset count years

cnt_mnths = 1 ! count of good months (months without missing data)

do $\bar{i} v=13,132$ ! year loop, starting on July 11999 and ending early (2009)

if (pyaat_sum(i,ii,cnt_yrs) .eq. -9999. .and. tval(i,ii,iv).1t.100) then pyaat_sum $\left(i, i i, c n t \_y r s\right)=0$

pyaat_sum $\left(i, i i, c n t \_y r s\right)=$ pyaat_sum(i,ii,cnt_yrs $)+t v a l(i, i i, i v)$

cnt_mnths $=$ cnt_mnths +1 ! update count of good months

else if (pyaat_sum(i,ii,cnt_yrs) .ne. -9999. .and. tval(i,ii,iv).1t.100) then

pyaat_sum(i,ii,cnt_yrs $)=$ pyaat_sum(i,ii,cnt_yrs $)+t v a l(i, i i, i v)$ else

cnt_mnths $=$ cnt_mnths +1 ! update count of good months

! do nothing

end if

if $(\bmod (i v, 12) \cdot e q . \theta)$ then

if (pyaat_sum(i,ii,cnt_yrs).ne.-9999) then

pyaat $\left(\bar{i}_{1} i i_{1} \text { cnt_yrs }\right)^{-}=$pyaat_sum $\left(i, i i_{1}\right.$ cnt_yrs $) / c n t \_m n t h s$ ! to get annual averages 
end if

cnt_yrs $=$ cnt_yrs +1 ! update count years

end if

cnt_mnths $=1$ ! reset count months

end do

end do ! end time loop

end do

!---Calculate prior year annual average temperature (winter taxa; start in July)

pyaat_sum_adj $=-9999$.

pyaat_adj $=-9999$.

do $i=1, n x \quad$ ! Loop over all longitudes

do $i \mathrm{i}=1$, ny ! Loop over all latitudes

cnt_yrs = 1 ! reset count years

cnt_mnths $=1$ ! count of good months (months without missing data)

do $\overline{i v}=7,126$ ! year loop, starting on July 11999 and ending early (2009)

if (pyaat_sum_adj(i,ii,cnt_yrs) .eq. -9999. .and. tval(i,ii,iv).lt.100) then

pyaat_sum_adj $\left(i, i i, c n t \_y r s\right)=0$

pyaat_sum_adj $\left(i, i i, c n t \_y r s\right)=$ pyaat_sum_adj(i,ii,cnt_yrs $)+$ tval $(i, i i$, iv)

cnt_mnths $=$ cnt_mnths +1 ! update count of good months end if

if (pyaat_sum_adj(i,ii,cnt_yrs) .ne.-9999. .and. tval(i,ii,iv).1t.100)

then

pyaat_sum_adj(i,ii,cnt_yrs $)=$ pyaat_sum_adj(i,ii,cnt_yrs $)+$ tval( $i, i i$, iv)

cnt_mnths $=$ cnt_mnths +1

end if

if $(\bmod (i v, 6) . e q \cdot \theta \cdot$ and. $\bmod (i v, 12) . n e \cdot 0)$ then ! add in extra conditional

if (pyaat_sum_adj( $\left.\left.i, i i, c n t \_y r s\right) . n e .-9999.\right)$ then

pyaat_adj $\left(\bar{i}, i i, c n t \_y r s\right)=$ pyaat_sum_adj $\left(i, i i, c n t \_y r s\right) / c n t \_m n t h s$ !

end if to get annual averages

cnt_yrs $=$ cnt_yrs +1 ! update count years

cnt_mnths $=1$ ! reset count months

end if

end do

end do ! end time loop

end do

end if ! on/off - calculate pyaat from monthy temperature data

!---Calculate Pollen Emissions Start Day of Year, end day of year and Duration-------

if (.true.) then

do $i=1$, nspec

if(i.eq.2 .or. i.eq.4 .or. i.eq.7) then !Alnus, Cupressaceae, Pinus $\operatorname{sDOY}(:,:,:, i)=\operatorname{NINT}\left(\left(\operatorname{sDOY} \_m(i) *\right.\right.$ pyaat_adj $\left.\left.(:,:,:)\right)+\operatorname{sDOY} b(i)\right)$

$\operatorname{eDOY}(:,:,:, i)=\operatorname{NINT}\left(\left(\right.\right.$ eDOY_m$_{-}(i) *$ pyaat_adj $\left.(:,:,:)\right)+$ eDOY_b $\left.(i)\right)$

! $\quad \operatorname{write}(*, *) \operatorname{sDOY}(69,74,:, i), \operatorname{dur}(6 \overline{9}, 74,:, i)$

else

$\operatorname{sDOY}(:,:,:, i)=\operatorname{NINT}((\operatorname{sDOY} m(i) *$ pyaat $(:,:,:))+\operatorname{sDOY} b(i))$

$\operatorname{eDOY}(:,:,:, i) \quad=\operatorname{NINT}\left(\left(\operatorname{eDOY}_{-} \mathrm{m}(\mathrm{i}) * \operatorname{pyaat}(:,:,:)\right)+\mathrm{eDOY} \_b(i)\right)$

Page 9 of 16 
end if

$\operatorname{dur}(:,:,:, i)=\operatorname{eDOY}(:,:,:, i)-\operatorname{sDOY}(:,:,:, i)$ !calculate duration from

do $i \mathrm{i}=1, \mathrm{nx}$

do $i i i=1$, ny

do $i v=1$, nyr

if(i.eq.2 .or. i.eq.4 .or. i.eq.7) then

if (pyaat_adj(ii,iii,iv) .1t. -9998) then

$\operatorname{sDOY}(i \bar{i}, i i i, i v, i)=-9999$.

$\operatorname{dur}(i i, i i i, i v, i)=-9999$.

$\operatorname{eDOY}(i i, i i i, i v, i)=-9999$.

else

end if

if (pyaat(ii,iii,iv) .lt. -9998) then

$\operatorname{sDOY}(i i, i i i, i v, i)=-9999$.

$\operatorname{dur}(i i, i i i, i v, i)=-9999$.

$\operatorname{eDOY}(i i, i i i, i v, i)=-9999$.

end if

end if

if (dur(ii,iii, iv,i).le.0) then

!write $(*, *)$ 'duration is zero or negative'

$\operatorname{dur}(i i, i i i, i v, i)=-9999$. end if

end do

end do

end do

end do

end if

! Due to memory array issues, calculate pollen in species loop, then write netCDF output for each species type

!---Output new netcdf file containing pollen count data for each grid cell for each day (2003-2010)---

! netcdf-output write parameters

start $=(/ 1,1,1,1 /)$

! count $=(/ \mathrm{nx}, \mathrm{ny}, \mathrm{ndy}, \mathrm{ny} r, 1 /) \quad$ ! note that will set start in the loop below count $=(/ \mathrm{nx}, \mathrm{ny}, \mathrm{nlev}$, ndytot $/)$

! dimension and variable attributes for netcdf-output

longname $(1)=$ 'longitude'

longname $(2)=$ 'latitude'

longname $(3)=$ 'level index'

longname $(4)=$ 'time'

!longname $(5)=$ 'pollen type'

longname $(5)=$ 'pollen emission potential $(f l u x) '$

units $(1)=$ 'degrees_east'

units $(2)=$ 'degrees_north'

units $(3)=$ 'level'

!units $(4)=$ 'days since 2006-12-31 00:00:00' ! set at beginning of code for convenience!

units $(5)=$ 'grains $m-2$ day -1 '

! Output NetCDF file create

rcode $=$ nf90_create $($ path $=$ "/good_share $/ \mathrm{mcwoz} /$ emis_out $/$ 
pollen_emissions_2001-2011_25km_speciated-calc_FINAL.nc",cmode = nf90_64 bit_offset, initialsize $=0$, ncid = ncidout $)$

! NetCDF file variable definition

rcode $=$ nf90_redef(ncidout)

rcode $=$ nf90_def_dim(ncidout, 'jx', count $(1), \operatorname{dimid} 1)$

rcode $=$ nf90_def_dim(ncidout, 'iy', count (2), dimid2)

rcode $=$ nf90_def_dim(ncidout,'lev', count (3), dimid3)

rcode $=$ nf90_def_dim(ncidout,'time', $\operatorname{count}(4)$, dimid4)

rcode $=$ nf90_def_dim(ncidout, 'spec', nspec, dimid5)

rcode $=$ nf90_def_dim(ncidout, 'year', nyr, dimid6)

if $($ rcode $/=$ nf90_noerr) write $(*, *)$ "netcdf error $=$

", $\operatorname{trim}\left(n f 90 \_\right.$strerror(rcode) )

!write $(*, *)$ "error after define dim"

id_2d(1) = dimid1

id_2d $(2)=$ dimid2

rcode = nf90_def_dim(ncidout, 'lon', id_2d,dimid6)

rcode $=$ nf90_def_dim(ncidout, 'lat', id_2d,dimid7)

rcode $=$ nf90_def_dim(ncidout, 'spec', nf90_unlimited, dimid5)

! $\operatorname{write}(*, *)$ dimid1, dimid2, dimid3, dimid4

! The dimids array is used to pass the dimids of the dimensions of

! the netCDF variables. Both of the netCDF variables we are creating

! share the same four dimensions. In Fortran, the unlimited

! dimension must come last on the list of dimids.

dimids $=(/$ dimid1, dimid2, dimid3, dimid4/)

phen_dimids $=(/$ dimid1,dimid2,dimid6, dimid5/)

phen_count $=(/ \mathrm{nx}, \mathrm{ny}, \mathrm{nyr}, \mathrm{nspec} /)$

! mcwoz edits: changed output variables to those of RegCM chemical emissions

format
rcode $=$ nf90_def_var(ncidout, 'lon'
rcode $=$ nf90_def_var(ncidout, ' lat'
,nf90_real,id_2d, lonoutid)
rcode $=$ nf90_def_var (ncidout, 'lev'
,nf90_real,id_2d, latoutid)
!rcode $=$ nf90_def_var(ncidout, ' day'
,nf90_real,dimid3, levoutid)
rcode $=$ nf90_def_var(ncidout, 'time'
,nf90_real,dimid3, dayoutid)
rcode $=$ nf90_def_var(ncidout, 'spec'
,nf90_real,dimid4, timeoutid)
rcode $=$ nf90_def_var(ncidout, 'year'
,nf90_real,dimid5, specoutid)
!rcode $=$ nf90_def__var(ncidout, 'yr'
,nf90_real, dimid6, yroutid)
!rcode $=$ nf90_def_var (ncidout, 'nspec'
,nf90_real, dimid4, yroutid)
!rcode $=$ nf90_def_var(ncidout, 'pc'
,nf90_real, dimid5, speoutid)
Ircode $=$ nf90 def-var(ncidout, 'POLLEN', nf90_real, dimids, pcoutid)
rcode $=$ nf90_def_var $\left(\right.$ ncidout $,{ }^{\prime} c l m \_l c ', n f 90 \_$double, (/dimid1, dimid2/), lcoutid)
rcode $=$ nf90_def_var (ncidout, 'sDOY', nf90_real, phen_dimids, sdoyoutid)
rcode $=$ nf90_def_var (ncidout, 'eDOY', nf90_real, phen_dimids, edoyoutid)
rcode = nf90_def_var(ncidout, 'dur',nf90_real, phen_dimids, duroutid)
rcode $=$ nf90_def_var(ncidout, 'DBL_POLL' ,nf90_real,dimids, dbloutid)
rcode = nf90_def_var(ncidout, 'ENL_POLL', nf90_real, dimids, enloutid)
rcode = nf90_def_var(ncidout, 'GRA_POLL', nf90_real, dimids, graoutid)
rcode $=$ nf90_def_var(ncidout, 'RAG_POLL',nf90_real, dimids, ragoutid)
!rcode $=$ nf90_def_.var(ncidout, 'clm_lc', nf90_double, $(/$
dimid1, dimid2/), polloutid)
!rcode $=$ nf90_def_var(ncidout, 'sDOY' , nf90_real, (/ 
dimid1, dimid2, dimid3, dimid4/), sdoyoutid)

if(rcode $/=$ nf90_noerr) write $(*, *)$ "netcdf error $=$

$", \operatorname{trim}\left(n f 90 \_s t r e r r o r(r c o d e)\right)$

!write $(*, *)$ "error after define var"

rcode $=$ nf90_put_att (ncidout, lonoutid, 'long_name', $\operatorname{trim}($ longname (1)))

rcode = nf90_put_att (ncidout, latoutid, 'long_name' ', trim(longname (2) ))

!rcode = nf90_put_att (ncidout, dayoutid, 'long_name', trim(longname (3)) )

!rcode = nf90_put_att (ncidout,yroutid, 'long_name', trim(longname (4)))

!rcode = nf90_put_att (ncidout, speoutid, 'long_name', trim(longname(5)))

!rcode = nf90_put_att (ncidout, pcoutid, 'long_name', trim(longname(6)))

rcode $=$ nf90_put_att (ncidout, timeoutid, 'long_name', $\operatorname{trim}($ longname(4)))

rcode $=$ nf90_put_att (ncidout, levoutid, 'long_name', trim(longname(3)))

!rcode $=$ nf90_put_att (ncidout, polloutid, 'long_name', $\operatorname{trim}($ longname (5)))

rcode = nf90_put_att (ncidout,dbloutid, 'long_name', trim(longname(5)))

rcode $=$ nf90_put_att (ncidout, enloutid, 'long_name', $\operatorname{trim}($ longname(5)))

rcode $=$ nf90_put_att (ncidout,graoutid, 'long_name', $\operatorname{trim}($ longname (5)))

rcode $=$ nf90_put_att (ncidout, ragoutid, 'long_name' ', trim(longname (5)))

if(rcode /= nf90_noerr) write $(*, *)$ "netcdf error $=$

", $\operatorname{trim}($ nf90_strerror(rcode) )

!write $(*, *)$ "error after put att long_name"

rcode $=$ nf90_put_att(ncidout, lonoutid, 'units', trim(units (1)))

rcode $=$ nf90_put_att (ncidout, latoutid, ' units ', $\operatorname{trim}($ units (2)) )

!rcode $=$ nf90_put_att (ncidout, dayoutid, 'units', trim(units(3)))

!rcode $=$ nf90_put_att (ncidout,yroutid, 'units', trim(units(4)))

! rcode $=$ nf90_put_att (ncidout, speoutid, 'units', trim(units (5)))

!rcode = nf90_put_att (ncidout,pcoutid, 'units', trim(units(6)))

rcode $=$ nf90_put_att (ncidout, timeoutid, 'units', trim(units (4)))

rcode $=$ nf90_put_att (ncidout, levoutid, 'units', trim(units (3)))

!rcode = nf90_put_att (ncidout,polloutid, 'units', trim(units(5)))

rcode $=$ nf90_put_att (ncidout, dbloutid, 'units', $\operatorname{trim}($ units (5)))

rcode $=$ nf90_put_att (ncidout, enloutid, 'units', $\operatorname{trim}($ units (5)) )

rcode $=$ nf90_put_att (ncidout,graoutid,' 'units', trim(units (5)))

rcode $=$ nf90_put_att (ncidout, ragoutid, 'units', trim(units (5)))

if(rcode $/=$ nf90_noerr) write $(*, *)$ "netcdf error $=$

", trim(nf90_strerror(rcode))

!write $(*, *)$ "error after put att units"

rcode $=$ nf90_enddef(ncidout) ! end definition mode

! NetCDF file data addition, basic dimension information

!rcode $=$ nf90_put_var $($ ncidout, dayoutid, idy, $\operatorname{start}(3: 3), \operatorname{count}(3: 3))$

!rcode = nf90_put_var (ncidout, yroutid, iyr, $\operatorname{start}(4: 4), \operatorname{count}(4: 4))$

rcode $=$ nf90_put_var (ncidout, lonoutid, $x$ lon, $\operatorname{start}(1: 2), \operatorname{count}(1: 2))$

rcode $=$ nf90_put_var (ncidout, latoutid, $x$ lat, $\operatorname{start}(1: 2), \operatorname{count}(1: 2))$

rcode $=$ nf90_put_var (ncidout, timeoutid,idy, $\operatorname{start}(4: 4), \operatorname{count}(4: 4))$

rcode $=$ nf90_put_var(ncidout, levoutid, $(/ 1 /), \operatorname{start}(3: 3), \operatorname{count}(3: 3))$

rcode $=$ nf90_put_var(ncidout, specoutid, $(/(i, i=1$, nspec $) /), \operatorname{start}(4: 4)$,

phen_count $(4: 4))$

rcode $=$ nf90_put_var(ncidout,yroutid,iyr, start $(3: 3)$, phen_count $(3: 3))$

rcode $=$ nf90_put_var $($ ncidout, lcoutid,frac_area $(:,:, 13)+$ frac_area $(:,:, 14)$,

start $(1: 2)$, count $(1: 2))$

rcode = nf90_put_var (ncidout, sdoyoutid, $\operatorname{sDOY},(/ 1,1,1,1 /)$, phen_count $)$

rcode = nf90_put_var(ncidout, sdoyoutid, $\operatorname{sDOY},(/ 1,1,1,1 /)$, phen_count $)$

rcode $=$ nf90_put_var (ncidout, edoyoutid, eDOY, $(/ 1,1,1,1 /)$, phen_count $)$ 
rcode = nf90_put_var(ncidout,duroutid,dur, $(/ 1,1,1,1 /)$, phen_count $)$

if (rcode $/=$ nf90_noerr) write $(*, *)$ "netcdf error $=$

", $\operatorname{trim}($ nf90_strerror(rcode) )

!---Calculate pollen count-----

wid $=3$ ! mcwoz edit: gaussian width (makes control of the model's width easier)

$! p c=0$.

$\mathrm{dbl}{ }_{\text {_poll }}=\theta$.

enl_poll $=\theta$.

gra_poll $=\theta$.

rag_poll $=0$.

write $(*, *)$ 'starting emissions calculation loop...'

do iv2 $=1$, nspec ! -2 cuts off the grasses

plac_flag $=$.true. ! write out which PFT this taxon's emissions will be added to

cnt $=1$ ! now counting days for total period of emissions

! initialize all values to zero

$! \mathrm{pc}=0 . \quad$ ! reinitialize for next count, comment out if doing sum

write $(*, *)$ 'Calculating taxon...', iv2, name(iv2)

do $i=1, n x$

!write $(*, *)$ 'X coord ', i

do $\mathrm{ii}=1$, ny

!write $(*, *)$ 'Y coord ', ii

cnt $=1$

do iv = yrst, yrend

!write $(*, *)$ 'Year ', iv

if (year(iv) .eq. '2004' .or. year(iv) .eq. '2008') then

else ndy $=366$

ndy $=365$

end if

tot_flx $=0$

if (dur(i,ii,iv, iv2).gt.0) then

!write $(*, *)$ 'duration is greater than zero, writing emissions'

if(.false. .and. iv.lt.nyr) then

mu1 $=\operatorname{sDOY}(i, i i, i v, i v 2)+\operatorname{dur}(i, i i, i v, i v 2) / 2$.

$m u 2=(\operatorname{ndy}+\operatorname{sDOY}(i, i i, i v+1, i v 2))+\operatorname{dur}(i, i i, i v+1, i v 2) / 2$.

sigma1 $=\operatorname{dur}(i, i i, i v, i v 2) /$ wid

sigma2 $=\operatorname{dur}(i, i i, i v+1, i v 2) /$ wid

do $j j=1$, ndy

tot_flx $=$ tot_flx $+\exp (-(\operatorname{real}(j j)-\operatorname{mu} 1) * * 2 . /(2 . * \operatorname{sigma} 1 * * 2))+$ $\exp (-($ real $(j j)-\operatorname{mu} 2) * * 2 . /(2 . * \operatorname{sigma} 2 * * 2))$

end do

ef_d = ef(iv2)/tot_flx ! daily emissions flux is division of annual emission factor over total yearly flux

else

$m u=\operatorname{sDOY}(i, i i, i v, i v 2)+\operatorname{dur}(i, i i, i v, i v 2) / 2$.

sigma $=\operatorname{dur}(i, i i, i v, i v 2) /$ wid

do $j j=1$, ndy

end do

tot_flx $=$ tot_flx $+\exp (-(r e a l(j j)-m u) * * 2 . /(2 . * s i g m a * * 2))$

end if

ef_d $=$ ef(iv2)/tot_flx 
do $i$ ii $=1$, ndy

write $(*, *)$ iv2, i, ii, iv, iii

if(.false. .and. iv.lt.nyr) then

!write $(*, *)$ 'negative sDOY. calculating double gaussian.'

gphen $=\exp (-($ real $($ iii $)-\operatorname{mu} 1) * * 2 . /(2 . * \operatorname{sigma1} * * 2))+\exp (-($ real $($ iii $)-$ mu $2) * * 2 . /(2 . * \operatorname{sigma} 2 * * 2))$

! pc(i,ii, iv, iii) = ef(iv2)*frac_area(i,ii,iv2)*gphen

if (iv2.eq.4.or. iv2.eq.7) then! ENL taxa

if(plac_flag) write $(*, *)$ 'adding to ENL PFT, negative sDOY'

if(plac_flag) write $(*, *)$ 'Year ', iv

enl_poll (i,ii,1,cnt) = enl_poll(i,ii,1,cnt) + ef_d*frac_area $(i$, $i i, i v 2) * g p h e n$

end if

if (iv2.eq.13 .or. iv2.eq.14) then ! grass taxa

if(plac_flag) write $(*, *)$ 'adding to grass PFT, negative sDOY'

if(plac_flag) write $(*, *)$ 'Year ', iv

gra_poli $(i, i i, 1, c n t)=\operatorname{gra} p \operatorname{poll}(i, i i, 1, c n t)+$ ef_d*frac_area $(i$, $i i, i v 2) * g$ phen

end if

if (iv2.eq.15) then ! ragweed

if(plac_flag) write $(*, *)$ 'adding to ragweed PFT, negative sDOY'

if(plac_flag) write $(*, *)$ 'Year ', iv

rag_poll $(i, i i, 1, c n t)=\operatorname{rag}(p o l l(i, i i, 1, c n t)+$ ef_d*frac_area $(i$,

end if ii, iv2)*gphen

if (iv2.eq.1 .or. iv2.eq.2 .or. iv2.eq.3 .or. iv2.eq.5 .or. iv2.eq. 6 .or. iv2.eq.8 .or. iv2.eq.9 .or. iv2.eq.10.or. iv2.eq.

11 .or. iv2.eq.12) then! DBL taxa

if(plac_flag) write $(*, *)$ 'adding to DBL PFT, negative sDOY'

if(plac_flag) write $(*, *)$ 'Year ', iv

dbl_poll $(i, i i, 1, c n t)=d b l \_p o l l(i, i i, 1, c n t)+$ ef_d*frac_area $(i$,

end if ii, iv2)*gphen

plac_flag $=$.false.

! pc(i, ii, 1, cnt $)=p c(i, i i, 1, c n t)+$ ef_d*frac_area( $i, i i, i v 2) * g p h e n * v f$

if(i.eq.67.and.ii.eq.74) then ! test for high CUPR grid cell write $(*, *)$ iv, iii, ef(iv2), frac_area(i, ii, iv2), gphen,

pc(i, ii, iii, iv)

endif

else

gphen $=\exp (-($ real $($ iii $)-m u) * * 2 . /(2 . * \operatorname{sigma} * * 2))$

! PFT-level pollen counts

!write $(*, *)$ 'positive sDOY. calculating single gaussian.'

if (iv2.eq.4 .or. iv2.eq.7) then ! ENL taxa

if(plac_flag) write $(*, *)$ 'adding to ENL PFT'

if(plac_flag) write $(*, *)$ 'Year ', iv

enl_poli $(i, i i, 1, c n t)=e n 1 \_p o l l(i, i i, 1, c n t)+$ ef_d*frac_area $(i, i i$, iv2) *gphen

end if

if (iv2.eq.13 .or. iv2.eq.14) then! grass taxa

if(plac_flag) write $(*, *)$ 'adding to grass PFT'

if(plac_flag) write $(*, *)$ 'Year ', iv

gra_poli $(i, i i, 1, c n t)=\operatorname{gra} p \operatorname{poll}(i, i i, 1, c n t)+$ ef_d*frac_area $(i$, $i i, i v 2) * g$ phen 
end if

if (iv2.eq.15) then ! ragweed

if(plac_flag) write $(*, *)$ 'adding to ragweed PFT'

if(plac_flag) write $(*, *)$ 'Year ', iv

rag_poll $(i, i i, 1, c n t)=\operatorname{rag}$ _poll $(i, i i, 1, c n t)+$ ef_d*frac_area $(i$,

end if $i \mathrm{i}, \mathrm{i} v 2) * g$ phen

if (iv2.eq.1 .or. iv2.eq.2 .or. iv2.eq.3 .or. iv2.eq.5 .or. iv2.eq. 6 .or. iv2.eq.8 .or. iv2.eq.9 .or. iv2.eq.10.or. iv2.eq.11.or. iv2.eq.12) then! DBL taxa

if(plac_flag) write $(*, *)$ 'adding to DBL PFT'

if(plac_flag) write $(*, *)$ 'Year ', iv

dbl_polil $(i, i i, 1, c n t)=d b l \_p o l l(i, i i, 1, c n t)+$

end if ef_d*frac_area $(i, i i, i v 2) * g$ phen

plac_flag $=$.false.

! pc(i, ii, iv, iii) = ef(iv2)*frac_area(i,ii, iv2)*gphen

! pc(i, ii, 1, cnt $)=p c(i, i i, 1, c n t)+$ ef_d*frac_area(i,ii, iv 2$) * g p h e n * v f$ end if ! if iv.lt.nyr for emissions calculation and placement

cnt $=$ cnt +1 ! update the day for $\mathrm{pc}$

! if(pc(i, ii, iv, iii) .eq. sqrt(-1) ) then

! $p c(i, i i, i v, i i i)=0$

! write $(*, *)$ "I have changed nan to zero."

! end if

enddo !day loop

end if ! if duration not equal to zero

enddo ! year loop

enddo ! latitude loop

enddo ! longitude loop

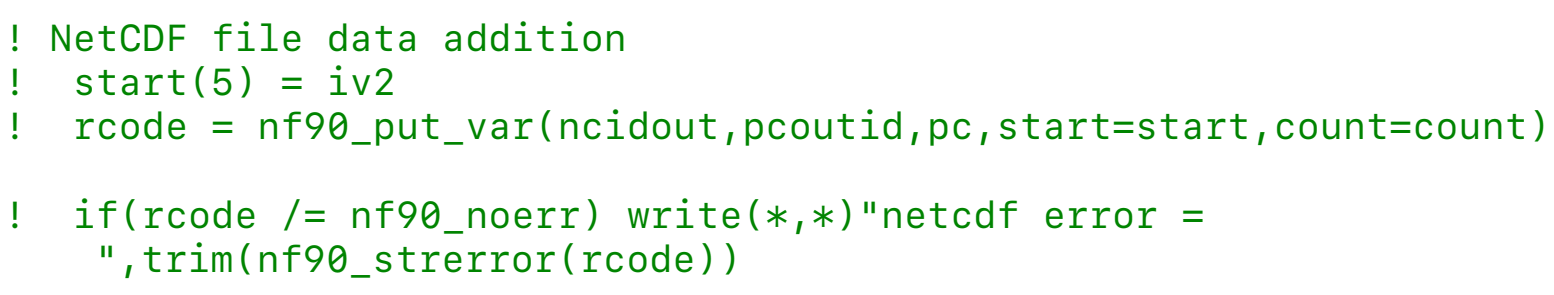

end do

! end iv2 species loop

!rcode = nf90_put_var(ncidout,polloutid,pc, start=start, count=count)

rcode = nf90_put_var(ncidout, dbloutid, dbl_poll, start=start, count=count)

rcode = nf90_put_var (ncidout, enloutid, enl_poll, start=start, count=count)

rcode = nf90_put_var (ncidout, graoutid,gra_poll, start=start, count=count)

rcode = nf90_put_var (ncidout, ragoutid, rag_poll, start=start, count=count $)$

! start1 $=(/ 1,1,1,1 /)$

! count1 $=(/ \mathrm{nx}, \mathrm{ny}, 1, \mathrm{nspec} /)$

!rcode = nf90_put_var(ncidout, sdoyoutid, sDOY, start=start1, count=count1)

if(rcode /= nf90_noerr) write $(*, *)$ "netcdf error $=$

", $\operatorname{trim}($ nf90_strerror(rcode) )

rcode $=$ nf90_close (ncidout)

end program PECM

Page 15 of 16 
Page 16 of 16 